Aus der Abteilung Klinische Neurophysiologie

(Prof. Dr. med. W. Paulus)

im Zentrum Neurologische Medizin

der Medizinischen Fakultät der Universität Göttingen

\title{
Überprüfung der Effektivität schwacher transkranieller Gleichstromstimulation bei Patienten mit chronischen Schmerzen
}

\author{
INAUGURAL - DISSERTATION \\ zur Erlangung des Doktorgrades \\ der Medizinischen Fakultät \\ der Georg - August - Universität zu Göttingen \\ vorgelegt von \\ Stefanie Kühnl \\ aus \\ Lutherstadt Wittenberg
}

Göttingen 2012 
D e k a n: Prof. Dr. med. M. P. Schön

I. Berichterstatterin: Prof. Dr. rer. nat. A. Antal

II. Berichterstatter: Priv. - Doz. Dr. med. F. Petzke

Tag der mündlichen Prüfung: 14.11.2012 


\section{Inhalt}

1 Einleitung $\quad 7$

$\begin{array}{lll}1.1 & \text { Schmerzentstehung } & 7\end{array}$

1.2 Schmerzweiterleitung 9

$\begin{array}{lll}1.3 & \text { Schmerzformen } & 12\end{array}$

$\begin{array}{lll}1.4 & \text { Schmerztherapie } & 13\end{array}$

$\begin{array}{lll}1.5 & \text { Transkranielle Magnetstimulation } & 17\end{array}$

1.6 Transkranielle Gleichstromstimulation 17

2 Stand der Forschung: Stimulation in der Schmerztherapie 22

$3 \quad$ Fragestellung und Ziel 25

4 Material und Methoden 26

$\begin{array}{lll}4.1 & \text { Patientenauswahl } & 26\end{array}$

$\begin{array}{lll}4.2 & \text { Studiendesign } & 28\end{array}$

4.3 Transkranielle Gleichstromstimulation 29

$\begin{array}{lll}4.4 & \text { Visuelle Analogskala } & 31\end{array}$

4.5 Fragebogen zur Erfassung von Nebenwirkungen 32

4.6 Statistische Analyse 32

4.7 Datenschutz und Genehmigung durch die Ethik Kommission 33

5 Ergebnisse $\quad 34$

5.1 Schmerzempfinden / VAS - Werte 34

$\begin{array}{lll}5.2 & \text { Nebenwirkungen } & 37\end{array}$

6 Diskussion $\quad 41$

$\begin{array}{lll}\text { 6.1 Schmerzreduktion } & 41\end{array}$

6.2 Elektrodengröße / Elektrodenposition 42 
$\begin{array}{lll}6.3 & \text { Nebenwirkungen } & 43\end{array}$

6.4 Placebo - Effekt 44

6.5 Klinische Relevanz 45

7 Zusammenfassung $\quad 48$

8 Literaturverzeichnis 50

9 Anhang 62

9.1 Aufklärungsbogen zur Teilnahme an der Studie 62

$\begin{array}{lll}9.2 & \text { Schmerztagebuch / Schmerzskala } & 67\end{array}$

9.3 Fragebogen zur Erfassung von Nebenwirkungen 68 


\title{
Abkürzungen
}

\author{
Abb.: Abbildung
}

ANOVA: Varianzanalyse (analysis of variance)

ATP: Adenosintriphosphat

CGRP: Calcitonin Gene - Related Peptide

COX: Cyclooxygenase

EEG: Elektroenzephalographie

M1: primär motorischer Kortex / Motorkortex

MCS: Motorkortex - Stimulation

MEP: motorisch evozierte Potentiale

MRT: Magnetresonanztomographie

NAS: numerische Analogskala

NGF: Nerve Growth Factor

NMDA: N - Methyl - D - Aspartat

NO: Stickstoffmonoxid

NSE: neuronenspezifische Enolase

PET: Positronenemissionstomographie

(r)TMS: (repetitive) Magnetstimulation

tDCS: transkranielle Gleichstromstimulation

Tab.: Tabelle

V5: visueller Kortex (extrastriatal, mediotemporal)

VAS: visuelle Analogskala

v.a.: vor allem

VEP: visuell evozierte Potentiale 
vs.: versus

z.B.: zum Beispiel

ZNS: zentrales Nervensystem 


\section{Einleitung}

Sowohl bei akutem, aber vor allem bei chronischem Schmerz kommt es zu einer Reihe von neuroplastischen Veränderungen im zentralen Nervensystem. Im Unterschied zu anderen Zellen des Körpers sind Neurone nicht zur Zellteilung fähig, sodass geschädigte Zellen untergehen und nicht ersetzt werden können. Jedoch besteht die Möglichkeit, dass die Aufgabe untergegangener Neurone durch andere Neurone übernommen wird. Diese Neuroplastizität bezeichnet die anhaltende morphologische oder funktionelle Veränderung des zentralen Nervensystems (ZNS) in Form von neuronaler Konnektivität, Repräsentationsmuster oder Nervenzelleigenschaften (Donoghue 1995).

Nicht immer stellt diese Neuroplastizität einen Vorteil dar: So kommt es z.B. zu einer verringerten Hemmung von Schmerzsignalen und Ausbildung eines Schmerzgedächtnisses, die als eine Ursache chronischen Schmerzes angesehen wird. Vereinfacht: Trotz fehlendem Schmerzreiz kommt es zur Aussendung permanenter Schmerzimpulse an das Gehirn, sodass dieses eine Art Schmerzgedächtnis entwickelt.

Chronischer Schmerz ist somit als eigenständige Krankheit, von der nach Angaben der Deutschen Gesellschaft für Schmerztherapie aktuell 15 Millionen Menschen betroffen sind, anzusehen. Die Folgen sind nicht nur für den einzelnen Patienten, sondern für die gesamte Gesellschaft spürbar: So kommt es zu verlängerten Klinikaufenthalten, verspäteter Wiedereingliederung, zu langfristig vermehrter Arbeitsunfähigkeit und vorzeitigem Ruhestand (Schäfer und Stein 1997). Folglich ist eine effektive Therapie bei der langfristigen Schmerzbekämpfung essentiell.

\subsection{Schmerzentstehung}

Die International Association for the Study of Pain definiert den Schmerz als "ein unangenehmes Sinnes- oder Gefühlserlebnis, das mit 
tatsächlicher oder drohender Gewebeschädigung einhergeht oder von den betroffenen Personen so beschrieben wird, als wäre eine solche Gewebeschädigung die Ursache“ (http://www.iasppain.org/AM/Template.cfm?Section=Pain_Defi...isplay. cfm\&ContentID=1728).

Im Rahmen einer Gewebeschädigung, welche durch thermische (z.B. Hitze oder Kälte), mechanische (z.B. Druck, Durchtrennung) oder chemische (z.B. Toxine) Reize entsteht, werden sogenannte Botenstoffe (Neurotransmitter) wie Adenosintriphosphat (ATP), Kalium - Ionen, Arachidonsäure und Sauerstoff - Radikale von den geschädigten Zellen freigesetzt. Diese führen zur Freisetzung der Enzyme Cyclooxygenase $1+2(\operatorname{COX} 1+2)$ in den Endothelzellen und in den einwandernden Leukozyten, welche die von den Nozizeptoren freigesetzte Arachidonsäure in Prostaglandin E2 umwandeln. Zudem werden aus den Endothelzellen Kinine ausgeschüttet und in Bradykinine umgewandelt. Neben Leukozyten und Endothelzellen sind auch Mastzellen an der Schmerzentstehung beteiligt: Sie setzen im Rahmen der Degranulation u.a. Histamin frei. Diese freigesetzten Botenstoffe bezeichnet man als Entzündungsmediatoren, die über die Produktion von Stickstoffmonoxid (NO) zur Vasodilatation führen, sodass ein lokales Ödem entsteht. Im Bereich des Ödems gelingt es nun den Leukozyten leichter, ins geschädigte Gewebe vorzudringen. Zusätzlich führen diese Schmerzmediatoren zur Erregung spezifischer Rezeptoren (Schmerzrezeptoren). Diese befinden sich fast überall im Körper und sind als Enden von Nervenfasern der Klasse $C$ und $A \delta$ in der Lage, Gewebeschädigungen wahrzunehmen und über ihre Nervenfaser an das Gehirn weiterzuleiten. 90\% dieser Schmerzrezeptoren (auch Nozizeptoren) sind in der Haut, aber auch in den inneren Organen, lokalisiert. Die Aktivierung der Nozizeptoren führt zur Ausschüttung von Substanz P, Somatostatin, Nerve Growth Factor (NGF) und Calcitonin Gene - Related Peptide (CGRP). Als Folge dessen werden weitere Schmerzrezeptoren in der Umgebung aktiviert, sodass der Schmerzreiz verstärkt wird. Der NGF 
bewirkt ein Wachstum der Nervenfasern vor allem in das umliegende Gewebe, um auch dieses schmerzempfindlicher zu machen.

\subsection{Schmerzweiterleitung}

Die von den Schmerzmediatoren aktivierten Nozizeptoren leiten die Schmerzimpulse - je nach Faserart sehr schnell oder langsam - über das Rückenmark an das Gehirn weiter. Die C - Fasern stellen dabei die langsamere Faserart dar, da sie von keiner Myelinscheide umgeben sind $(0,5-2 \mathrm{~m} / \mathrm{s})$. Die $A \delta$ - Fasern sind von einer Myelinscheide umgeben und leiten somit deutlich schneller $(5-25 \mathrm{~m} / \mathrm{s})$.

Beide Faserarten laufen in den Spinalganglien (erstes Neuron) zusammen. Von dort ziehen sie als zentripetaler Fortsatz des Spinalganglions (primäre nozizeptive Afferenz) aufwärts und durch den lateralen Teil der Hinterwurzel in das Rückenmark ein.

Bereits auf Ebene des Rückenmarks kommt es durch die sogenannte Reflexverschaltung zur ersten Reaktion auf den Schmerzreiz: Ein Teil der über das Hinterhorn eintreffenden, von den Spinalganglien stammenden Fasern zieht zum Vorderhorn und ermöglicht somit ohne bewusste Wahrnehmung des Reizes eine sofortige Reaktion auf diesen.

Die übrigen Fasern gabeln sich im Tractus dorsolateralis (Lissauer Trakt) auf und enden im dorsalen Bereich der Substantia gelatinosa und im Hinterhorn des Rückenmarks. Die primären Fasern steigen auf der ipsilateralen Seite des Rückenmarks nach oben, wohingegen die sekundären Fasern im Bereich der Commissura alba auf die Gegenseite kreuzen, im zweiten Neuron umgeschaltet werden und als Tractus spinothalamicus lateralis aufwärts ziehen. Hierbei ist der Tractus nicht als festes Faserbündel zu verstehen, sondern als lockeres Gefüge, dem sich auf dem Weg nach zentral weitere Fasern anderer Systeme und verschiedener Wurzelhöhen jeweils ventromedial anschließen. 
Im Bereich der Medulla oblongata laufen die Fasern am lateralen Rand über die Olive (Lemniscus spinalis):

Ein Teil der Fasern zieht als Tractus spinothalamicus in den Thalamus und die übrigen Fasern als Tractus spinoreticularis zur Formatio reticularis. Die Aktivierung der Fasern des Tractus spinoreticularis führt zur Steigerung der Aufmerksamkeit, da dieser Bestandteil des sogenannten aufsteigenden Aktivierungssystems ist, bei dessen Erregung der Organismus in einen absoluten Wachzustand versetzt wird. Die spinothalamischen Fasern ziehen weiter zum Thalamus im Mittelhirn und schließen sich in dorsolateraler Position dem Lemniscus medialis an. Ein Großteil der Fasern endet dort im Bereich des Nucleus ventralis posterior thalami (drittes Neuron) in somatotroper Anordnung und in den übrigen Thalamuskernen. Der andere Teil läuft weiter bis in die Postzentralregion des Kortex, welche sich als Endstation der sensiblen Fasern im Gyrus postcentralis befindet (Kahle 2002, 324).

Die (motorische) Reaktion auf Schmerzreize erfolgt über die absteigenden (efferenten) Bahnen der Willkürmotorik - den Tractus corticospinalis (Pyramidenbahn) und die Fibrae corticonucleares. Über diese kann der Kortex die subkortikalen motorischen Zentren kontrollieren und entweder dämpfend bzw. hemmend oder aktivierend wirken. So werden über diese Bahnen ständig tonische Erregungen geleitet, um rasche und plötzliche Bewegungen zu fördern. Zwei Drittel der Fasern der Pyramidenbahn stammen aus der Region des Gyrus praecentralis und ein Drittel entstammt dem Gyrus postcentralis. Sie verlaufen durch die Capsula interna und bilden mit den kortikopontinen Bahnen am Übergang zum Mittelhirn die Pedunculi cerebri, wobei die Pyramidenbahnfasern mittig lokalisiert sind. In Höhe der Medulla oblongata enden die Fibrae corticonucleares in den jeweiligen Hirnnervenkernen. 70-90\% der Fasern der Pyramidenbahn kreuzen in der Decussatio pyramidum auf die Gegenseite und ziehen als Tractus corticospinalis lateralis ab- 
wärts zum Rückenmark. Die nicht gekreuzten Fasern ziehen im Tractus corticospinalis anterior zum Rückenmark und kreuzen erst im Bereich der Commissura alba auf die Gegenseite.

Im Bereich der Zona intermedia zwischen Vorder- und Hinterhorn enden die meisten Fasern an Zwischenneuronen. Nur wenige Fasern, vor allem die zu den distalen Extremitätenabschnitten gehörigen, reichen bis zur motorischen Vorderhornzelle und können somit durch Pyramidenbahnimpulse in Form einer Aktivierung der Flexoren und einer Hemmung der Extensoren gezielt kontrolliert werden. Der Anteil der Parietallappenbahnen endet in den Hinterstrangkernen (Nucleus gracilis und Nucleus cuneatus) sowie in der Substantia gelatinosa des Hinterhorns und reguliert die eintreffenden sensiblen Erregungen.

Nach Umschaltung auf Zwischenneurone erfolgt die Weiterleitung der Impulse auf die Vorderhornzellen. Von dort ziehen wiederum die motorischen Nervenfasern zu der jeweiligen Zielmuskulatur und verzweigen sich in dieser (Kahle 2002, 308). Dabei trifft auf jede Muskelfaser ein Axonzweig, sodass die Gruppe von Muskelfasern, die vom Axon einer Vorderhornzelle ( $\alpha$ - Motoneuron) versorgt wird, als motorische Einheit bezeichnet wird und sich bei Erregung geschlossen kontrahiert. Im Bereich der Axonaufzweigungen liegen Zellkerne von sogenannten Schwannzellen, die die Axone vor ihren Endigungen umhüllen. Unter diesen wiederum liegen Zellkerne von Muskelfasern der motorischen Endplatte. Die knopfartigen Auftreibungen der Axone reichen in die motorische Endplatte und setzen bei Erregung an der präsynaptischen Membran den Botenstoff Acetylcholin aus ihren Vesikeln frei. Dieses entleert sich in den synaptischen Spaltraum zwischen Axonendigung und Muskelfaser und führt so zur Depolarisation der Muskelfasermembran, sodass eine Kontraktion im Muskel ausgelöst wird (Kahle 2002, 312). 


\subsection{Schmerzformen}

Bezüglich der Schmerzdauer unterscheidet man zwischen zwei Formen - akutem und chronischem Schmerz:

Der akute Schmerz ist ein plötzlich auftretendes, akutes Signal für eine Schädigung und hat somit zumeist eine klar erkennbare Ursache, der entgegengewirkt werden kann, sodass er zeitlich begrenzt ist und nach dem Akutereignis wieder abklingt (z.B. Schmerzen bei Entzündungen, Knochenbrüchen, Nieren- und Gallenkoliken).

Schmerz, der jedoch länger als drei Monate und somit länger als der zu erwartende Heilungsprozess anhält, wird als chronisch bezeichnet und hat seine eigentliche Warnfunktion verloren (z.B. Osteoporose, Tumorschmerzen, Phantomschmerzen).

Aufgrund der Entität gibt es eine Unterteilung in nozizeptiven, neuropathischen und psychogenen Schmerz:

Nozizeptiver Schmerz beschreibt Schmerzen, deren Ursache in einer Reizung der Schmerzrezeptoren liegt. Werden Schmerzrezeptoren an der Oberfläche (z.B. bei einer Schürfwunde) stimuliert, wird dieser am Ort der Schädigung wahrgenommen (Oberflächenschmerz). Gewebeschädigung, die hingegen in der Tiefe zu einer Reizung der Schmerzrezeptoren führt (z.B. bei einer Divertikulitis), wird oftmals auf die Körperoberfläche projiziert. Diesen Schmerz bezeichnet man dann als Übertragungsschmerz, zu dem auch der sogenannte von den inneren Organen ausgehende Viszeralschmerz zählt.

Neuropathischer Schmerz ist als Eigenschmerz der Nerven zu verstehen. Dieser ist oftmals brennenden oder stechenden Charakters und wird wiederum in die Neuralgie (Eigenschmerz eines Nerven in dessen Versorgungsgebiet, z.B. Trigeminusneuralgie), die Neuropathie (Schmerz durch längerfristige Schädigung eines oder mehrerer Nerven, z.B. Polyneuropathie), den Phantomschmerz (Schmerz durch blind en- 
dende Nervenendigungen in abgetrennten Gliedmaßen, z.B. nach Unterschenkelamputation) und die Kausalgie (Schmerz durch direkte Nervenschädigung, z.B. Schnittverletzung) unterschieden (Steinbach und Diener 2007).

Die Diagnose eines psychogenen Schmerzes sollte erst nach Ausschluss einer organischen Genese gestellt werden. Diese Form des Schmerzes ist auf nicht verarbeitete, psychische Konflikte zurückzuführen. In der Regel ist den Betroffenen der Zusammenhang zwischen den Schmerzen und psychischen Konflikten nicht bewusst und nur schwer erkennbar, sodass zur Klärung dieser Konflikte oft viel Zeit und Vertrauen aufgebracht werden müssen.

\subsection{Schmerztherapie}

Ziel der Schmerztherapie ist es, eine Schmerzregredienz oder linderung für den Betroffenen zu erreichen. Dabei ist das Zusammenspiel medikamentöser / invasiver Maßnahmen und nichtmedikamentöser Maßnahmen sowie die Aufklärung des Patienten, dessen aktive Mitarbeit und die Auseinandersetzung mit dem Krankheitsbild essentiell.

Die medikamentöse Therapie erfolgt nach dem Stufenschema der Weltgesundheitsorganisation (WHO). Diese umfasst die Behandlung in drei Stufen:

1.Stufe: Nicht - Opioid - Analgetika, welche schmerzstillend, fiebersenkend und teilweise auch entzündungshemmend wirken (z.B. Ibuprofen, Acetylsalicylsäure, Metamizol, Diclofenac). Sollte durch Therapie mit diesen Präparaten keine ausreichende Linderung erzielt werden, können sie mit Mitteln der Stufe zwei kombiniert werden, da beide unterschiedliche Wirkmechanismen aufweisen. 
2.Stufe: Schwache Opioide, welche in ihrer Wirkung dem Morphin ähneln (z.B. Tilidin, Codein, Tramadol). Sollte auch nach Applikation der Höchstdosis keine Schmerzreduktion eintreten, wird die Therapie auf Mittel der Stufe drei umgestellt.

3.Stufe: Starke Opioide, welche auch über einen längeren Zeitraum in höheren Dosen eingenommen werden können (z.B. Fentanyl, Morphin). Neben den Nicht - Opioid- und Opioid - Analgetika gibt es die Klasse der Antidepressiva, der Antiepileptika und andere Substanzklassen, die in der Schmerztherapie Anwendung finden:

Antidepressiva, vor allem trizyklische Antidepressiva (z.B. Amitriptylin), finden durch inre analgetische Wirkung in Form einer Aktivierung absteigender schmerzinhibitorischer Bahnen eine häufige Anwendung in der Therapie chronischer oder neuropathischer Schmerzen, wobei diese Wirkung unabhängig von der antidepressiven oder sedierenden Komponente auftritt (Max et al. 1987; Onghena und van Houdenhove 1992). Weniger effektiv, jedoch bei auftretenden Nebenwirkungen im Rahmen einer Therapie mit trizyklischen Antidepressiva zum Einsatz kommend, sind Serotonin - Wiederaufnahmehemmer (z.B. Citalopram, Fluoxetin, Paroxetin) (Mc Quay et al. 1996; Max et al. 1992).

Antiepileptika (z.B. Gabapentin, Pregabalin, Lamotrigin, Carbamazepin) kommen wegen ihrer hohen Wirksamkeit vor allem bei einschießenden oder getriggerten neuropathischen Schmerzen zum Einsatz (Mc Quay et al.1995; Wiffen et al. 2001). Carbamazepin hat sich in der Behandlung der Trigeminusneuralgie etabliert. Lamotrigin erwies sich in der Therapie zentraler Schmerzen nach einem Hirninfarkt und auch im Rahmen einer diabetischen Polyneuropathie als besonders effizient (Eisenberg et al. 2001; Vestergaard et al. 2001). Gabapentin und Pregabalin hingegen werden bei den übrigen chronischen Schmerzsyndromen angewandt, nachdem ihre Wirksamkeit in großen Studien bestätigt werden konnte (Rowbotham et al. 1998; Backonja et al. 1998; 
Dworkin et al. 2003). Ihr Wirkmechanismus ist wie der des Lamotrigin durch die Blockade spannungsabhängiger Natriumkanäle zu erklären.

Zu den übrigen Substanzklassen zählen die Benzodiazepine, welche häufig bei begleitenden Schlafstörungen unter chronischen Schmerzen angewandt werden, jedoch aufgrund ihrer Toleranz- und Abhängigkeitsentwicklung sowie Entzugssymptomatik nur in geringem Maß Einsatz finden sollten. Auch Neuroleptika sollten nicht vordergründig und nur in Kombination verabreicht werden, da es bisher keine Evidenz für deren analgetische Wirkung gibt. Stattdessen sollten diese bei unruhigen, agitierten Patienten zur Sedierung oder unter Opioid - Therapie als Antiemetikum eingesetzt werden (Metaanalyse: Nix 1998). Schmerzen im Rahmen entzündlicher Erkrankungen wird häufig mit Kortikosteroiden (v.a. Prednisolon) entgegengewirkt. Auch hier sind eigenständige analgetische Effekte bisher nicht nachgewiesen (Saag et al. 1996). Lediglich der Einsatz in der Malignomtherapie zeigt eine Besserung der Schmerzen allerdings nur über sekundäre Effekte wie einer Regredienz der Tumorschwellung und somit auch der Nervenkompression sowie einer Steigerung des Wohlbefindens durch die appetitanregende und Übelkeit mindernde Wirkung.

Die nichtmedikamentösen Therapieverfahren umfassen:

- physikalische Maßnahmen: Im Rahmen der Schmerztherapie hat sich der Einsatz von Wärme aufgrund der krampflösenden, entspannenden und durchblutungsfördernden Wirkung als hilfreiche Unterstützung bei nicht entzündlicher Genese herausgestellt. Neben der Wärmetherapie sind auch andere Verfahren wie z.B. Balneotherapie, Kältetherapie, Elektrotherapie, Phototherapie und Mechanotherapie gute Reize, die zur Funktionserhaltung des menschlichen Organismus beitragen.

- Physiotherapie: Verschiedene Methoden der Krankengymnastik sind in der erfolgreichen Schmerztherapie unerlässlich. Hierbei werden günstige, physiologische Bewegungsabläufe trainiert. Neben dem Mus- 
kelaufbau spielt auch die Dehnung der Muskulatur beim Erhalt der Gelenkbeweglichkeit eine wichtige Rolle. Weiterer Pluspunkt der Bewegung ist die Steigerung des Selbstwertgefühls und die Ablenkung der Konzentration vom Schmerz weg.

- psychologische Therapie: Eine begleitende psychologische Betreuung ist in der Behandlung chronischer Erkrankungen essentieller Bestandteil. Vor allem die kognitiv - behavioralen Verfahren haben sich in zahlreichen Studien als besonders effizient herausgestellt (Metaanalyse: Morley et al. 1999). Gerade bei depressiven und somatoformen Störungen empfiehlt sich eine psychiatrische Mitbetreuung, um dem Patienten eine Aufklärung, aktive Mitarbeit und Auseinandersetzung mit dem Krankheitsbild zu ermöglichen.

- Entspannungstherapie: Schmerzen verursachen Verspannungen, welche wiederum Schmerzen verursachen können. Deshalb ist die Entspannungstherapie wesentlicher Bestandteil der Schmerztherapie. Hierzu zählen aufgrund der guten Effizienz und raschen Erlernbarkeit die progressive Muskelrelaxation nach Jacobson (Rehfisch und Basler 1999), autogenes Training, Biofeedback (Kröner - Herwig 1999), Hypnose und operante Verfahren.

- Akupunktur: Hierbei werden eine Reihe von Akupunkturnadeln an definierten Punkten platziert und durch Drehen, Applikation von Hitze oder Strom stimuliert. Systematische Rückblenden konnten in den Studien von Smith et al. (2008) und van Tulder et al. (2001) bei Patienten mit chronischen Schmerzsyndromen und chronischen Rückenschmerzen keine Effizienz der analgetischen Wirkung der Akupunktur darstellen. Einzig in der Therapie der Gonarthrose ließ sich eine Überlegenheit der Akupunktur gegenüber der medikamentösen Standardtherapie eruieren (Endres et al. 2007).

Neben zahlreichen pharmakologischen, physikalischen und psychologischen Ansätzen haben sich in den vergangenen Jahrzehnten verschie- 
dene neuromodulatorische Verfahren etabliert, so z.B. die Stimulation des primär motorischen Kortex (M1) mittels transkranieller Gleichstromstimulation (tDCS) und repetitiver transkranieller Magnetstimulation (rTMS). Beide Verfahren erlauben durch Änderung der neuronalen Aktivität oder des Ruhemembranpotentials die Induktion und Modulation neuroplastischer Veränderungen im Großhirn.

\subsection{Transkranielle Magnetstimulation}

Die transkranielle Magnetstimulation (TMS) ist eine nicht invasive Methode, bei welcher mit Hilfe von Spulen, die auf der Kopfhaut platziert werden und welche Magnetfelder erzeugen, die Aktivität des Gehirns erhöht oder gehemmt wird. Im Gegensatz zu anderen, insbesondere elektrischen, Verfahren ist der Spulenreiz magnetisch, sodass keine elektrischen Widerstände der Haut, des Knochens oder der Hirnhäute überwunden werden müssen.

Die TMS verursacht dabei durch induzierte elektrische Felder an der Zellmembran Erregbarkeitsveränderungen, die auch über die Stimulationsdauer hinaus anhalten können. Diese Felder können in drei Varianten induziert werden: Einzelimpuls - TMS, repetitive niederfrequente und repetitive hochfrequente TMS (Siebner und Ziemann 2007).

Es erfolgt keine Erregung von Schmerzrezeptoren, sodass diese Therapiemethode in Abhängigkeit vom Stimulationsgebiet nur selten schmerzhaft ist. Nachteil dieser Methode ist die begrenzte Anwendbarkeit: So können beispielsweise Epileptiker bei Applikation hoher Frequenzen einen epileptischen Anfall erleiden oder bei Trägern von Herzschrittmachern Herzrhythmusstörungen ausgelöst werden.

\subsection{Transkranielle Gleichstromstimulation}

Bei der ebenfalls nicht invasiven Methode der transkraniellen Gleichstromstimulation (tDCS) werden Elektroden an der Kopfhaut ange- 
bracht und ein kontinuierlicher Gleichstrom verabreicht. Da die Elektroden nur auf der Kopfhaut anliegen und somit keinen direkten Kontakt zur Hirnrinde haben, kommt es zu einer Abschwächung der Stromdichte, sodass von der ursprünglich applizierten Stromdichte nur ca. 50\% die Hirnrinde erreichen (Rush und Driscoll 1968). Bereits seit den 1960er Jahren ist bekannt, dass auf diese Weise die Aufmerksamkeit und die Hirnleistung verändert werden können. Neben durchgeführten Versuchen am Tier (Bikson et al. 2004) gab es im Verlauf auch Untersuchungen an gesunden Probanden sowie an Patienten. Dabei erzeugt die tDCS eine prolongierte neuronale Erregbarkeits- und Aktivitätsänderung durch Veränderungen des neuronalen Membranpotentials in Richtung De- oder Hyperpolarisierung. Die unterschiedlichen Stimulationsparameter der tDCS stellen das Pendant zu Dosierung und Einnahmehäufigkeit im Rahmen einer medikamentösen Therapie dar. So sind in den vergangenen Jahren diverse Stimulationsparameter und deren Wirkung untersucht worden, z.B. die Elektrodenposition, die Elektrodengröße, die Stromstärke, die Stimulationsdauer und die Stimulationspolarität. So konnte nachgewiesen werden, dass die kortikale Exzitabilität in Abhängigkeit von der Stimulationspolarität (anodal vs. kathodal) erhöht oder vermindert wird (Zusammenfassung von Nitsche et al. 2008). Je nach Stimulationsdauer sind diese Veränderungen auch nach Beendigung der Stimulation anhaltend (Nitsche und Paulus 2000, 2001). Der primäre Effekt der tDCS besteht in einer De- oder Hyperpolarisation der neuronalen Membranpotentiale, bei dem die nachhaltige Wirkung, welche NMDA - Rezeptor - abhängig ist, variiert (Creutzfeld et al. 1962; Bindman et al. 1964; Liebetanz et al. 2002).

tDCS ändert nicht nur die Aktivität des Areals, über welchem die Elektrode platziert ist, sondern auch in entfernteren Arealen, am ehesten durch Verschaltung des hauptsächlich stimulierten Areals mit den benachbarten Strukturen (Lang et al. 2005). Diese Methode bietet darüber hinaus zuverlässige Bedingungen bezüglich einer Placebo - Stimulation, um die Spezifität des Effektes zu beurteilen. 
Doch nicht nur im Bereich des Motorkortex, sondern auch in nichtmotorischen Hirnarealen wurde die Effektivität der tDCS nachgewiesen: Antal et al. (2006) überprüften die Veränderungen des visuellen Kortex nach tDCS. Es wurden die N70 - Potentiale im Rahmen der visuell evozierten Potentiale gemessen. Nach anodaler Stimulation konnte eine erhöhte Amplitude und nach kathodaler Stimulation eine Reduktion der Amplitude im VEP beobachtet werden. Auch zehn Minuten nach Stimulation waren diese Unterschiede signifikant. In einer weiteren Studie von Antal und Nitsche (2004) konnte eine verbesserte visuomotorische Koordination durch Verbesserung der Bewegungsperzeption nach tDCS - Stimulation über V5 festgestellt werden. Probanden wurden dabei zu einem Zeichnungstest aufgefordert, welchen die Probanden nach anodaler Stimulation erfolgreicher absolvierten als die Probanden der Gruppe der kathodalen Stimulation.

2008 konnten Chaieb et al. eine Änderung der Exzitabilität im visuellen Kortex nachweisen: Die Ergebnisse zeigten einen nachhaltigen Effekt der kathodalen Gleichstromstimulation für weitere zehn Minuten nach Applikation. Jedoch konnten im Rahmen der kathodalen Stimulation keine Unterschiede zwischen den Geschlechtern eruiert werden. Nach der anodalen Stimulation hingegen ließ sich bei den weiblichen Probanden im Vergleich zu den männlichen eine signifikante Steigerung der kortikalen Exzitabilität für weitere zehn Minuten nach Applikation nachweisen. Unmittelbar nach der Stimulation war dieser Unterschied nicht nachzuweisen.

Boros et al. (2008) überprüften nach Stimulation des prämotorischen Kortex eine Ausbreitung der Neuroplastizität auf den ipsilateralen M1 über die kortikokortikalen Verbindungen. 16 Probanden erhielten eine anodale und kathodale Stimulation über dem prämotorischen Kortex, acht Probanden eine Stimulation des dorsolateralen präfrontalen Kortex. Lediglich die anodale Stimulation des prämotorischen Kortex zeigte in den anschließenden TMS - Protokollen eine kurze intrakortikale Inhi- 
bition oder intrakortikale Bahnung, sodass eine Ausbreitung des tDCS Effektes auf benachbarte Hirnareale vermutet wurde.

In der Studie von Nitsche et al. (2010) wurde der Effekt von Serotonin auf die durch tDCS induzierte Neuroplastizität untersucht. Zwölf gesunde Probanden erhielten eine kathodale oder anodale Stimulation über M1 unter zeitgleicher Applikation einer Dosis von 20mg Citalopram oder Applikation eines Placebo - Medikaments. Die Ergebnisse der Veränderung der Exzitabilität und damit vermutete neuroplastische Veränderungen wurden durch ein TMS - Protokoll erfasst: In der Gruppe der Probanden mit der Placebo - Medikation konnte unter anodaler Stimulation eine Steigerung und unter kathodaler Stimulation eine Minderung der Exzitabilität für einen Zeitraum von 60-120 Minuten beobachtet werden. Die Gruppe mit der serotonergen Medikation wies eine Steigerung und Verlängerung der Exzitabilität unter anodaler und eine Umkehr der durch kathodale Stimulation erzeugten Inhibition in eine Bahnung auf.

Thirugnanasambandam et al. (2011) kombinierten die tDCS mit einer kurz andauernden willkürlichen Muskelkontraktion. Auch hierbei konnte anhand verschiedener TMS - Protokolle eine Veränderung der Exzitabilität nachgewiesen werden: Die Muskelkontraktionen reduzierten die durch anodale tDCS hervorgerufene Steigerung der Exzitabilität. Die im Rahmen der kathodalen tDCS auftretende Minderung der Exzitabilität wurde durch diese Mukselkontraktionen umgekehrt.

Auch auf Ebene der Kognition wurde die Effektivität der tDCS nachgewiesen: So fanden Kincses und Antal (2004) eine Leistungsverbesserung des implizierten Lernens bei gesunden Probanden nach Stimulation des präfrontalen Kortex. Ebenso widmeten sich Fregni et al. (2006c) den Veränderungen der kognitiven Fähigkeiten durch dieses nichtinvasive Verfahren. Sie zeigten, dass eine anodale Stimulation im Vergleich zur Placebostimulation zu keiner Verschlechterung der kognitiven Leistungen bei Patienten mit Depressionen führt, sondern die Leistungsfähigkeit des Arbeitsgedächtnisses steigert. Eine weitere Studie von 
Fregni et al. (2006d) wies die signifikante Reduktion von depressiven Symptomen nach der Applikation von tDCS in der Verumgruppe im Vergleich zur Placebogruppe nach. Stimuliert wurde hierbei der Bereich auf der Kopfhaut, der im EEG der Elektrode F3 entspricht. Die Auswertung erfolgte mit Hilfe der Hamilton - Rating - Skala für Depressionen und mittels des Beck - Depressions - Inventars.

Der Effekt der Neuroplastizität wurde auch durch Venkatakrishnan et al. (2011) nachgewiesen. In dieser Studie wurde die Ruheaktivität gesunder Probanden 35 Minuten vor und 35 Minuten nach der Verum- oder Placebo - Stimulation durch Magnetenzephalographie aufgezeichnet. Es konnte nach der Verumstimulation, nicht jedoch nach der Placebostimulation, im frontotemporalen Bereich eine Abnahme der Alpha- und eine Zunahme der Gammaaktivität beobachtet werden.

In einer Studie von Boggio et al. (2011) wurde die Neuroplastizität nach Applikation von tDCS im Rahmen der Alzheimer - Erkrankung angenommen. Es konnten eine Besserung der kognitiven Funktion hinsichtlich der Sprache und der Wiedererkennungsfunktion erreicht werden.

Die Perspektive, dass die Modulation kortikaler Erregbarkeit durch die tDCS bei der Behandlung neurologischer und auch psychiatrischer Erkrankung klinisch relevant sein wird, nimmt damit zunehmend Konturen an. Nicht zuletzt aufgrund der Tatsache, dass diese Methode bislang als sicher und nebenwirkungsarm gilt. Die Reizung der Kopfhaut führt zu einem von Probanden beschriebenen kurzzeitigen Gefühl des Kribbelns oder auch leichten Ziehens (Nitsche et al. 2008). Persistierende oder kortikale Schädigungen konnten - bei Einhaltung der Richtlinien bezüglich der Kontraindikationen und Stimulationsparameter - bisher auch mittels EEG, MRT (mit Kontrastverstärkung) und durch Messungen der NSE (Destruktionsmarker) nicht nachgewiesen werden, sodass die tDCS weiterhin als gut verträgliche und nebenwirkungsarme Methode anzusehen ist. 


\section{Stand der Forschung: Stimulation in der Schmerzthe- rapie}

Tsubokawa et al. (1993) zeigten als erste Forschungsgruppe, dass therapierefraktäre zentrale Schmerzen nach einem Schlaganfall durch Stimulation des M1 mittels epiduraler Elektroden reduziert werden können.

Es folgten weitere Studien zur Überprüfung der Effektivität der Motorkortex - Stimulation (MCS) bei anderen Erkrankungen, u.a. bei der Trigeminusneuralgie, in denen eine Schmerzreduktion nachgewiesen werden konnte (Meyerson et al. 1993).

Vielversprechende Ergebnisse konnten auch durch Wallace et al. (2004), die die Effektivität der tiefen Hirnstimulation erprobten, erbracht werden. Diese zählt jedoch zu den invasiven Methoden, denen die nicht invasiven Methoden wie TMS und tDCS gegenüberstehen.

Zunächst wurde die Effektivität der rTMS in den Arbeiten von Rollnik et al. (2002) als zu gering und nicht signifikant eingestuft, jedoch konnte in weiteren Studien eine erhebliche Schmerzreduktion bei Patienten mit chronisch neuropathischen Schmerzen nachgewiesen werden (Khedr et al. 2005; Lefaucheur et al. 2001, 2004).

Die tDCS wurde initial an Patienten mit einem Schmerz nach Trauma des Rückenmarks und bei an Fibromyalgie leidenden Patienten erprobt (Fregni et al. 2006a; Fregni et al. 2006b).

In einer Studie von Fregni et al. (2006a) wurden Patienten mit chronischen Schmerzen randomisiert und entweder mit einer Gleichstromstimulation über dem rechten oder linken primär motorischen Kortex (M1) (2mA für 20 Minuten an fünf aufeinander folgenden Tagen) oder mittels Placebo - Stimulation versorgt. Hierbei war eine signifikante Besserung der Schmerzen nach der anodalen Stimulation des Areals M1 zu eruieren, nicht jedoch nach der Placebo - Stimulation. Die Folgestudie mit 
einer Patientengruppe, deren Teilnehmer an Fibromyalgie leiden, wies eine vergleichbare Regredienz der Schmerzwahrnehmung auf (Fregni et al. 2006b).

Auch Valle et al. (2009) untersuchten die Schmerzregredienz mittels tDCS bei 41 Fibromyalgie - Patientinnen. Sie erhielten täglich an zehn aufeinanderfolgenden Tagen über 20 Minuten eine Gleichstromstimulation mit 2mA oder eine Placebo - Stimulation über dem linken M1 oder dem dorsolateralen präfrontalen Kortex. Die Ergebnisse zeigten, dass die Stimulation an beiden Orten zu einer Reduktion der Schmerzwahrnehmung und Besserung der Lebensqualität führte. Jedoch konnte nachgewiesen werden, dass nur die Stimulation über M1 zu einer langanhaltenden Schmerzreduktion (30 und 60 Tage nach Stimulation erfasst) führte.

2009 überprüften Fenton et al. die Anwendung der tDCS bei Patienten mit chronischen Beckenschmerzen. Sie erhielten an zwei aufeinanderfolgenden Tagen jeweils über 20 Minuten aktive oder Placebo - Stimulation. Hierbei konnte im Rahmen der aktiven Gleichstromstimulation eine Schmerzregredienz beobachtet werden.

Mori et al. (2010) führten in einer randomisierten, placebo - kontrollierten, doppelblinden Studie über einen Zeitraum von fünf Tagen eine anodale Gleichstrom- oder Placebo - Stimulationen durch. Patienten der anodalen Stimulationsgruppe berichteten von einer Schmerzreduktion, die der Placebo - Gruppe jedoch nicht. Auch hierbei wurde eine Langzeitwirkung in der Verumgruppe beobachtet, sodass auch hier von einer Erregbarkeitsveränderung ausgegangen wurde.

Die Effektivität der tDCS bei Patienten mit chronischer Migräne wurde von Dasilva et al. (2012) in einer randomisierten und placebo - kontrollierten Studie überprüft: 13 Patienten erhielten in zehn Sitzungen über vier Wochen eine Gleichstromstimulation mit 2mA. Hierbei wurde eine Reduktion der Schmerzintensität und der Dauer der Migräneepisode 
beobachtet. Auch hierbei konnte ein nachhaltiger Effekt nur in der Gruppe, die tatsächlich Gleichstrom erhielt, beobachtet werden.

Riberto et al. (2011) kombinierten die tDCS mit einem multidisziplinären Rehabilitationsprogramm. 23 Fibromyalgie - Patienten erhielten wöchentliche Sitzungen von anodaler oder Placebo - Stimulation, kombiniert mit einem Rehabilitationsprogramm. Im Vergleich zur Placebo Gruppe konnte in der Gruppe mit anodaler Stimulation jedoch nur eine Besserung der Lebensqualität nachgewiesen werden, die VAS - Werte blieben unverändert. 


\section{Fragestellung und Ziel}

Ziel dieser Arbeit war es, mittels der nicht invasiven Methode der schwachen transkraniellen Gleichstromstimulation, welche sich polaritätsspezifisch auf die kortikale Erregbarkeit auswirkt, eine Schmerzreduktion bei Patienten mit chronischen Schmerzen zu erreichen, welche auch nach der Intervention andauert.

Darüber hinaus sollen die Ergebnisse der Studie einen tieferen Einblick in die individuellen Mechanismen maladaptiver Neuroplastizität und Informationen zur Entwicklung von Strategien zur Behandlung neuropathischer Schmerzen bieten.

Im Vergleich zur Studie von Fregni et al. (2006a) variierten bei der hiesigen Arbeit vier Parameter:

1. Die Intensität der Stimulation betrug $1 \mathrm{~mA}$ (2mA bei Fregni et al. 2006a).

2. Es wurde eine einheitliche Größe der Elektroden gewählt ( $4 \times 4 \mathrm{~cm})$, um den Fokus der Stimulation zu spezifizieren $(5 \times 7 \mathrm{~cm}$ bei Fregni et al. 2006a).

3. Zusätzlich wurde ein cross - over - Design genutzt, welches $60 \%$ der Patienten einschließt; 13 Patienten erhielten beide Stimulationsarten.

4. Die möglichen intrakortikalen Effekte repetitiver tDCS über dem M1 Areal wurden mittels gepaarter Puls - Stimulation untersucht. Die gepaarte Puls - Stimulation beinhaltet verschiedene Protokolle, um die Veränderung der Exzitabilität des menschlichen Motorkortex durch Iokale Verschaltungen afferenter Impulse aus anderen Arealen des Gehirns zu prüfen (Valls - Sole et al. 1992; Kujirai et al. 1993).

Abschließend wurden die möglichen Nebenwirkungen durch einen Fragebogen, welcher von der Arbeitsgruppe der Abteilung Neurophysiologie entwickelt wurde, erfasst (Poreisz et al. 2007). 


\section{Material und Methoden}

\subsection{Patientenauswahl}

Die Probanden waren ambulante Patienten der Abteilung Klinische Neurophysiologie der Universität Göttingen sowie Patienten von niedergelassenen Ärzten. Die Patienten wurden als geeignet und teilnahmefähig eingestuft, wenn sie folgende Kriterien erfüllten:

1. Über die letzten sechs Monate bestehender chronischer Schmerz.

2. Eine Schmerzstärke von drei oder mehr auf der visuellen Analogskala (VAS) $(0=$ kein Schmerz, $10=$ stärkster vorstellbarer Schmerz $)$ im letzten Monat vor Beginn der Stimulation.

3. Therapierefraktäre Schmerzen, welche trotz Applikation von zwei Analgetika und / oder Narkoleptika - in adäquater Dosis - über sechs Monate anhielten.

Ausgeschlossen wurden Patienten, die Carbamazepin im Therapieregime führen, da dieses die Effekte der anodalen Stimulation bereits nach einer Sitzung beeinflussen kann (Liebetanz et al. 2002).

Außerdem wurden Patienten ausgeschlossen, welche an einer gravierend internistischen oder psychiatrischen Erkrankung leiden, eine weitere neurologische Erkrankung erleiden (akut oder in der Vorgeschichte), unter einer rezeptiven oder globalen Aphasie leiden, einen Substanzabusus in der Eigenanamnese aufweisen oder in den letzten acht Wochen vor Studieneinschluss an einer anderen klinischen Studie teilgenommen haben.

Des Weiteren mussten Patienten ausgeschlossen werden, welche metallische Materialien im Körper tragen (Herzschrittmacher, Akupunktur Nadeln, Granatsplitter), sich derzeit in der Schwangerschaft oder Stillperiode befinden und unter 18 beziehungsweise über 70 Jahre alt sind. 
Insgesamt nahmen 23 Patienten im Alter von 28-70 Jahren an der Studie teil, darunter befanden sich fünf männliche Teilnehmer. Die klinischen Angaben zu den Patienten sind in Tabelle 1 aufgeführt.

Die Studie war eine randomisierte, doppelblinde, placebo - kontrollierte und Einzelzentrumversuchsstudie zur Überprüfung der Effektivität von täglichen Sitzungen mit Verabreichung von Gleichstrom an fünf aufeinander folgenden Tagen bei Patienten mit chronischem Schmerz.

In 13 Fällen war das cross - over - Design erfült: Diese Patienten durchliefen nach der ersten Stimulationsperiode und einer anschließenden sechswöchigen Pause eine weitere Stimulationsperiode. Bedingung hierfür waren: Die VAS - Werte mussten mindestens zehn Tage vor erneuter Stimulation auf dem baseline - Level gewesen sein. Die übrigen zehn Patienten durchliefen die Stimulationsperiode einmalig; sechs davon die anodale Stimulation, vier die Placebo - Stimulation. Zwei von 23 Patienten (ein Patient aus der Patientengruppe mit einmaliger anodaler Stimulation und ein Patient aus dem cross - over - Design) mussten ausgeschlossen werden, da sie ihre Schmerztagebücher aus der Folgephase nach der Stimulation nicht zur Verfügung stellten.

Tab.1 Teilnehmerdaten

\begin{tabular}{|l|l|l|l|}
\hline & $\begin{array}{l}\text { anodale } \\
\text { tDCS }\end{array}$ & $\begin{array}{l}\text { Placebo- } \\
\text { tDCS }\end{array}$ & $\begin{array}{l}\text { anodale und Place- } \\
\text { bo-tDCS }\end{array}$ \\
\hline Anzahl & 6 & 4 & 13 \\
\hline Altersbereich in Jahren & $28-70$ & $50-70$ & $48-70$ \\
\hline
\end{tabular}

Tab.2 Ätiologie der Schmerzen

\begin{tabular}{|l|l|l|l|}
\hline & $\begin{array}{l}\text { anodale } \\
\text { tDCS }\end{array}$ & $\begin{array}{l}\text { Placebo- } \\
\text { tDCS }\end{array}$ & $\begin{array}{l}\text { anodale und Placebo- } \\
\text { tDCS }\end{array}$ \\
\hline Fibromyalgie & & 2 & 1 \\
\hline chronische Rückenschmerzen & 2 & 1 & 5 \\
\hline
\end{tabular}




\begin{tabular}{|l|l|l|l|}
\hline Trigeminusneuralgie & 2 & & 1 \\
\hline atypischer Gesichtsschmerz & & & 2 \\
\hline Arthrose & 1 & 1 & 2 \\
\hline Phantomschmerz & 1 & & \\
\hline Post - stroke Schmerz & & & 1 \\
\hline Polyneuropathie & & & 1 \\
\hline
\end{tabular}

Tab.3 Dauer der chronischen Schmerzen

\begin{tabular}{|l|l|l|l|}
\hline & anodale tDCS & Placebo-tDCS & $\begin{array}{l}\text { anodale und Placebo- } \\
\text { tDCS }\end{array}$ \\
\hline$>$ 5 Jahre & 5 & 3 & 6 \\
\hline zw. 2 und 5 Jahren & 1 & 1 & 5 \\
\hline$<2$ Jahre & 0 & 0 & 2 \\
\hline $\begin{array}{l}\text { baseline - VAS - Wert } \\
\text { (SD) }\end{array}$ & $7,11(1,2)$ & $7,0(1,5)$ & $5,8(2,1) ; 5,95(2,2)$ \\
\hline
\end{tabular}

Tab.4 aktuelle Schmerzmedikation

\begin{tabular}{|l|l|l|l|}
\hline & $\begin{array}{l}\text { anodale } \\
\text { tDCS }\end{array}$ & $\begin{array}{l}\text { Placebo- } \\
\text { tDCS }\end{array}$ & $\begin{array}{l}\text { anodale und Pla- } \\
\text { cebo-tDCS }\end{array}$ \\
\hline nicht steroidale Antiphlogistika & 2 & 2 & 2 \\
\hline Pregabalin & & 1 & 2 \\
\hline Amitriptylin & & 1 & \\
\hline Morphin & 2 & & 2 \\
\hline keine Medikamente & 2 & & 7 \\
\hline
\end{tabular}

\subsection{Studiendesign}

Die Studie bestand aus drei Abschnitten:

1. Eine vierwöchige Periode, in der die Patienten täglich ihr subjektives Schmerzempfinden anhand einer VAS in einem Schmerztagebuch dokumentierten (baseline - evaluation). 
2. Eine einwöchige Periode, in der die Patienten an fünf aufeinander folgenden Tagen täglich mit Placebo- oder anodaler tDCS stimuliert wurden.

3. Eine vierwöchige Folgeperiode, in der die Patienten täglich ihr subjektives Schmerzempfinden anhand einer VAS in einem Schmerztagebuch dokumentierten.

Bereits während der baseline - evaluation wurden die Patienten bezüglich der anodalen vs. Placebo - Stimulation randomisiert.

\subsection{Transkranielle Gleichstromstimulation}

Die Patienten nahmen in liegender Position auf einem Stuhl mit montierter Kopfstütze Platz. Die Stimulation wurde stets von derselben Person durchgeführt. Die Elektroden wurden in Schwammtäschchen (4x4cm über M1 und $5 \times 10 \mathrm{~cm}$ über der kontralateralen Orbita), welche wiederum in Kochsalzlösung getränkt wurden, verpackt. Mittels der Elektroden wurde der von einem batteriebetriebenen konstanten Gleichstromstimulator (NeuroConn, IImenau, Deutschland) produzierte Gleichstrom appliziert (siehe Abb. 3). Die Patienten erhielten entweder eine Placebo- oder eine anodale Stimulation über dem M1 - Areal (primär motorisches Areal) (siehe Abb. 1).

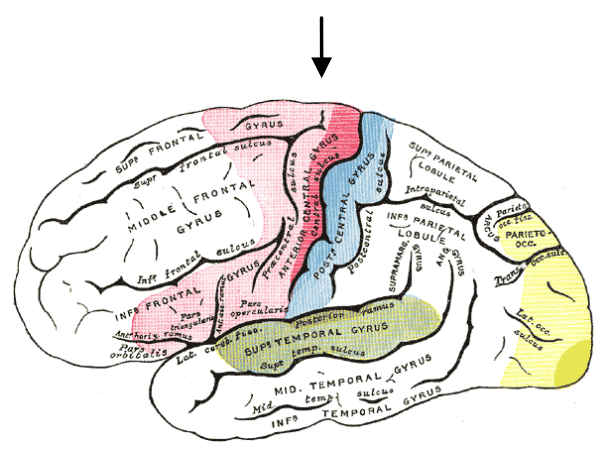

Abb.1 Hirnareal M1 - primär motorischer Kortex (siehe Pfeil) (http://de.academic.ru/pictures/dewiki/103/gray756.png) 
Für die Stimulation wurde die aktive Elektrode über dem linksseitigen Hirnareal, welches die Motorik der Hand repräsentiert, platziert. Die Referenzelektrode wurde dabei supraorbital auf der kontralateralen Seite platziert (unabhängig von der Seite des Schmerzes). Bezüglich der Exzitabilität des M1 - Areals erwies sich diese Elektrodenposition in vorangegangenen Studien als besonders effektiv (Nitsche und Paulus 2001) (siehe Abb. 2).

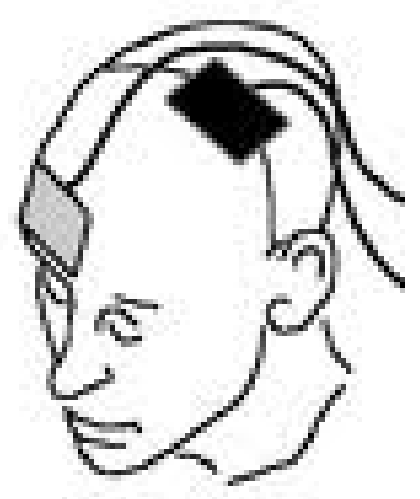

Abb.2 Platzierung der Elektroden

(http://nwg.glia.mdcberlin.de/en/courses/method/2010 /01.html)

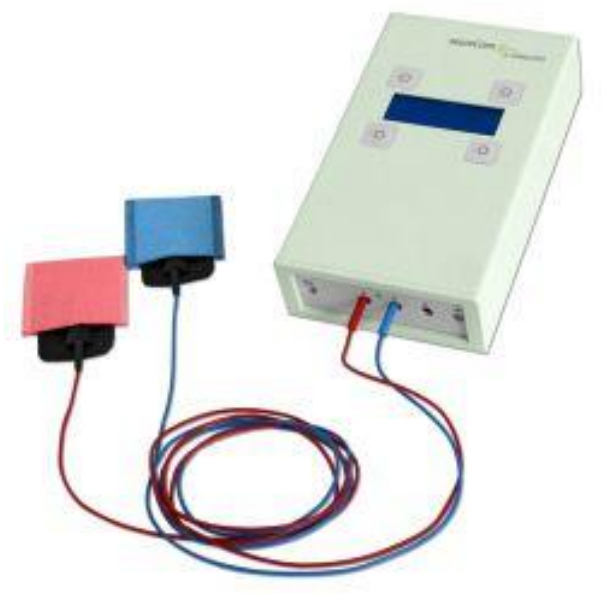

Abb.3 Gleichstromstimulator der Firma Neuroconn IIImenau

(http://www.neuroconn.de/de/lo esungen/dc-stimulator/)

Die Stimulation des M1 - Areals mittels tDCS induziert so weitreichende Änderungen der Aktivität kortikaler Areale und kann demzufolge auch die Aktivität des kontralateralen M1 - Areals verändern (Lang et al. 2005). Diese Erkenntnis lässt vermuten, dass unilaterale Behandlungen mit tDCS auch bei Patienten mit bilateral lokalisierten Schmerzen suffizient sein können. Da die Elektrode bei der tDCS groß ist, werden nicht nur der M1 stimuliert, sondern auch umgebende Hirnareale. 
Nach Platzierung der Elektroden wurde ein konstanter direkter Gleichstrom mit einer Intensität von $1 \mathrm{~mA}$ über 20 Minuten appliziert. Die Patienten spürten zu Beginn der Stimulation einen kurz andauernden ziehenden Schmerz über beiden Elektroden. Bei der Applikation von Placebo - Stimulation wurden die Elektroden ebenso und nach oben genannter Anordnung platziert. Auch hierbei war zu Beginn der Stimulation ein ziehender Schmerz über beiden Elektroden zu spüren, jedoch wurde der Gleichstromstimulator nach 30 Sekunden automatisch abgeschaltet, sodass die Patienten für die verbleibende Zeit keinen Gleichstrom verabreicht bekamen.

\subsection{Visuelle Analogskala}

Die Erfassung des subjektiven Schmerzempfindens erfolgte mittels einer visuellen Analogskala, welche über $10 \mathrm{~cm}$ reichte. Hierbei stellt jede Zentimetereinheit einen Wert von null bis zehn dar. Die Null bedeutet dabei, dass keine Schmerzen vorliegen und die Zehn repräsentiert den stärksten vorstellbaren Schmerz. Sowohl Validität als auch Reproduzierbarkeit dieser Art der Schmerzerfassung konnten in Studien demonstriert werden (Bolton und Wilkinson 1998).

Die Studienteilnehmer wurden gebeten ihr Schmerzempfinden 30 Tage vor Beginn der Stimulation, während der Stimulation und 30 Tage nach der Stimulation jeweils dreimal täglich zu erfassen. Wir instruierten die Patienten zur weiteren Einnahme ihrer aktuellen Medikation, wobei auch pflanzliche Präparate und Naturheilverfahren (wie z.B. Akupunktur und Massagen) erlaubt waren, wenn sie bereits vier Wochen vor der baseline angewandt und konstant während der Studie beibehalten wurden. 


\subsection{Fragebogen zur Erfassung von Nebenwirkungen}

Da potenzielle Nebenwirkungen der Gleichstromstimulation bisher nicht vollständig bekannt sind, erhielten die Patienten sowohl nach der anodalen als auch nach der Placebo - Stimulation einen Fragebogen (Poreisz et al. 2007).

Der Fragebogen beinhaltet Bewertungsskalen zum einen für die Präsenz und zum anderen für den Schweregrad von möglichen Nebenwirkungen wie Kopfschmerzen, Konzentrationsstörungen, akute Stimmungsveränderungen, visuelle Wahrnehmungsstörungen, Müdigkeit und unangenehme Sensationen wie Schmerz, Kribbeln, Brennen oder Jucken unter den Elektroden. Die Nebenwirkungen wurden während und im Anschluss an die fünftägige Stimulation erfasst. Die Inzidenz der Nebenwirkungen wurde in einem binären System kodiert, die Antwort nein wurde durch die Ziffer Null und die Antwort ja durch die Ziffer eins ersetzt. Die Ausprägung der jeweiligen Nebenwirkung wurde in einer numerischen Analogskala (NAS) von eins bis fünf dokumentiert, wobei die Ziffer eins eine milde Ausprägung und die Ziffer fünf eine extrem starke Ausprägung repräsentieren. Die Inzidenz und Ausprägung während und nach der Stimulation wurden im Anschluss getrennt voneinander betrachtet.

\subsection{Statistische Analyse}

Bezüglich der Schmerzwahrnehmung wurden zwei verschiedene Analysearten durchgeführt: Zunächst wurden alle teilnehmenden Patienten eingeschlossen, um die Konditionen der anodalen und Placebo - Stimulation gegenüberzustellen. Zum Zweiten wurden die cross - over - Analyse nutzend die Daten von zwölf Patienten ausgewertet. Um auszuschließen, dass Abweichungen zwischen beiden Gruppen auf deduktiven Unterschieden zwischen beiden Stimulationsgruppen beruhen, wurden die VAS - Werte beider Gruppen vor der Stimulation mittels des Student - $\mathrm{t}$ - Tests verglichen. 
Bei der Analyse der VAS - Werte stellte die VAS - Schmerzskala die abhängige Variable dar, wohingegen die Art der Stimulation (anodal vs. Placebo) und die Zeit (Durchschnittswerte zehn Tage vor der Stimulation, Tag eins bis fünf während der Stimulation und der siebte, 14., 21. und 28. Tag nach der Stimulation) die Einflussgrößen waren.

Des Weiteren wurden, wenn geeignet und unter Anwendung der Bonferroni Korrektur, post - hoc - Vergleiche zwischen Placebo- und anodaler Stimulation und zwischen der Evaluation nach der Stimulation und der baseline ausgeführt.

Darüber hinaus wurden für alle Endpunkte Student - $t$ - Tests angewandt, in denen nur die Zeit die konstante Größe darstellte.

Alle Ergebnisse wurden als Mittel- und Standardfehler angegeben; die statistische Signifikanz bezieht sich auf einen zweiseitigen $p$ - Wert < 0,05 .

\subsection{Datenschutz und Genehmigung durch die Ethik - Kommission}

Alle Probanden wurden vor Beginn der Stimulation in einem Gespräch aufgeklärt und gaben ihr schriftliches Einverständnis. Über etwaige Ausschlusskriterien (Alter $<18$ oder $>70$, Schwangerschaft / Stillperiode, Metallteile im Körper, andere neurologische Erkrankung, rezeptive oder globale Aphasie, gravierende internistische oder psychiatrische Erkrankung, Einnahme von Carbamazepin, Substanzabusus in der Eigenanamnese, Teilnahme an einer anderen klinischen Studie innerhalb von acht Wochen vor Studieneinschluss) erfolgte eine schriftliche Aufklärung (siehe Anhang).

Die Studie erfüllt die ethischen Standards der Helsinki - Deklaration von 1964 (World Medical Organization 1996) und wurde von der Ethik Kommission der Georg - August - Universität Göttingen genehmigt (Antragsnummer 27 / 12 / 06). 


\section{Ergebnisse}

\subsection{Schmerzempfinden / VAS - Werte}

In Bezug auf die baseline - VAS - Werte gab es keinen signifikanten Unterschied ( $t=1,8 ; p=0,19$ ) zwischen den Patienten der Placebogruppe und denen, die eine anodale Stimulation erhielten. Um festzustellen, ob eine Besserung der Schmerzen mit der Behandlung mit Gleichstrom assoziiert ist, führten wir wiederholte ANOVA - Messungen durch. Hierbei wurde die abhängige Variable gegen eine normalisierte VAS - Schmerzskala ausgetauscht, wohingegen die Zeit (baseline, erster, zweiter, dritter, vierter, fünfter Tag und folgende) und die Stimulationsart (anodal vs. Placebo) die unabhängige Variable darstellte. Die gesamte Patientengruppe betrachtend war ein signifikanter Effekt der Stimulation ( $F(1,32)=5,32 ; p<0,05)$ und der Zeit $(F(8,256)$ $=2,14 ; p<0,03)$ zu eruieren, jedoch keine Signifikanz bei der Interaktion zwischen beiden $(F(8,256)=0,28 ; p=0,9)$.

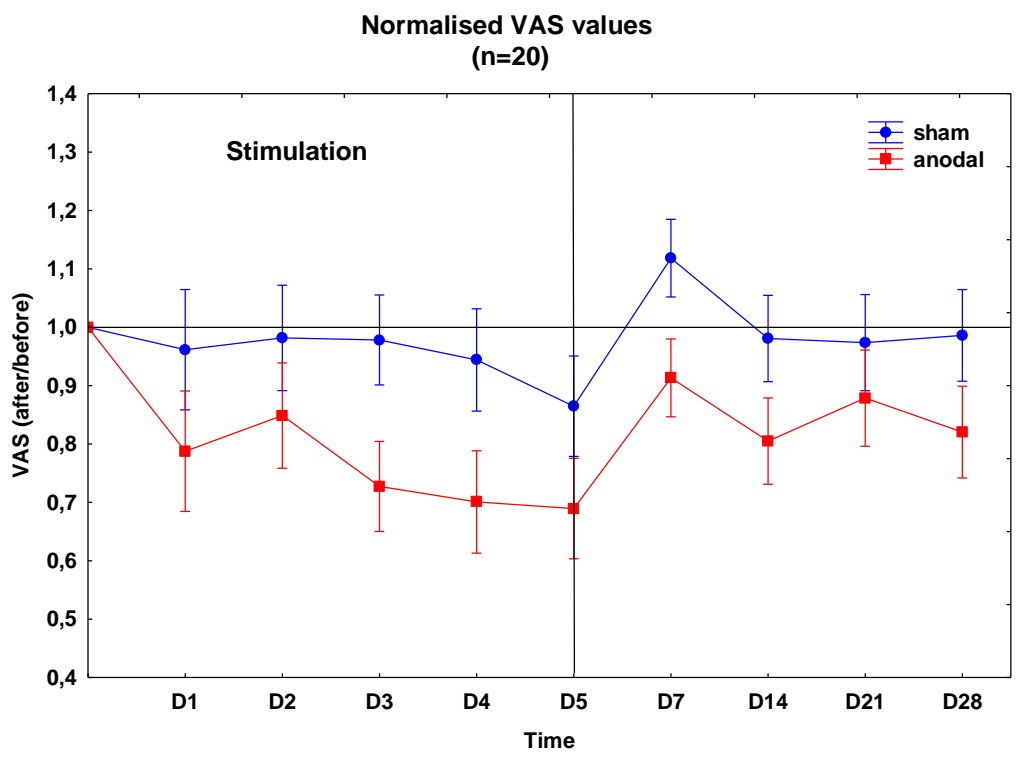

Abb.4 Schmerzwerte aller Patienten während und nach der Stimulation (normalisiert gegen die Ausgangs - VAS - Werte (Mittelwert der VAS - Werte von zehn Tagen vor der Stimulation) $($ sham $=$ Placebo, $\mathrm{D}=\mathrm{Tag}$, time $=$ Zeit $)$ 
Die Ergebnisse des Student - $t$ - Tests wiesen eine signifikante Differenz zwischen der anodalen und der Placebo - Stimulation am dritten und siebten Tag auf ( $p<0,05$; Tag 3: 27,3\% Schmerzreduktion bei anodaler Stimulation vs. 2,7\% Schmerzreduktion bei Placebo - Stimulation und Tag 7: 8,9\% Schmerzreduktion bei anodaler Stimulation vs. $11 \%$ Schmerzzunahme bei Placebo - Stimulation).

Bei Betrachtung der zwölf Patienten, welche beide Stimulationsarten durchliefen, zeigte sich nach der ANOVA - Analyse ein signifikanter Effekt in Bezug auf die Stimulationsart $(F(1,22)=14,3 ; p<0,05)$. Jedoch blieben hierbei die Zeit $(F(8,176)=1,24 ; p=0,27)$ und die Interaktion zwischen Zeit und Stimulationsart $(F(8,176)=0,35 ; p=0,9)$ ohne Signifikanz.

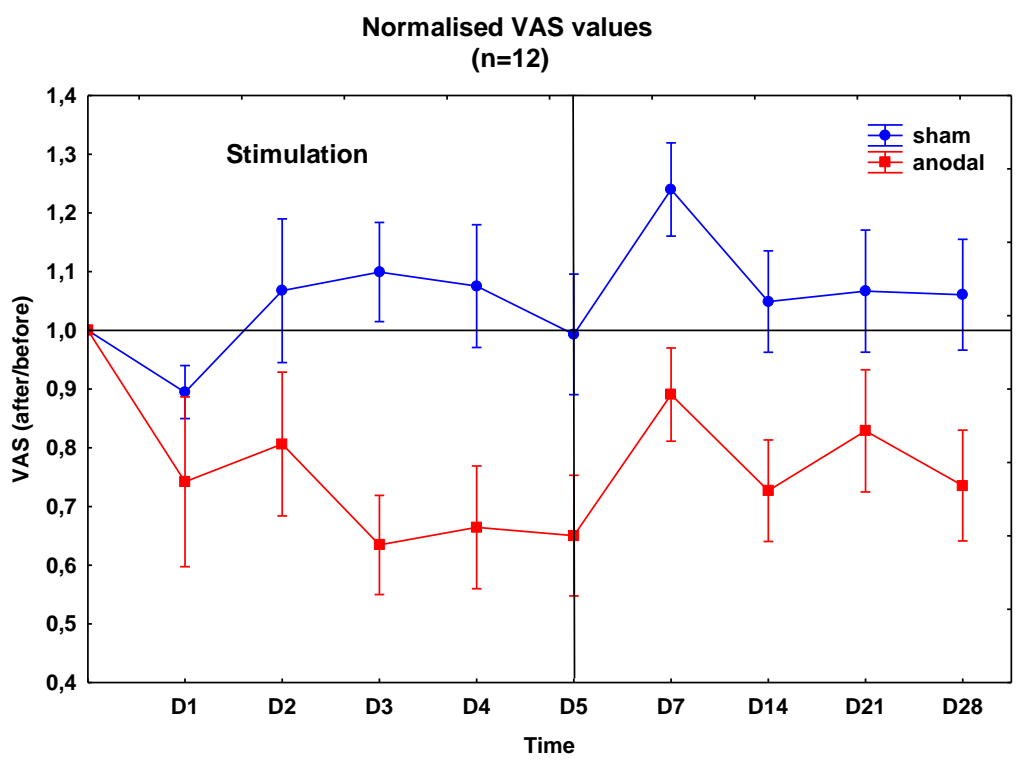

Abb.5 Schmerzwerte der zwölf cross - over - Patienten während und nach der Stimulation (normalisiert gegen die Ausgangs - VAS - Werte (Mittelwert der VAS - Werte von zehn Tagen vor der Stimulation); sham = Placebo, $D=$ Tag, time $=$ Zeit)

Der Student - $\mathrm{t}$ - Test erbrachte in dieser Patientengruppe einen signifikanten Unterschied zwischen der anodalen und Placebo - Stimulation am Tag drei, vier, fünf, sieben, 14 und 28 ( $p<0,05$; Tag 3: 36,5\% Schmerzreduktion bei anodaler Stimulation vs. $9 \%$ Schmerzzunahme 
bei Placebo - Stimulation; Tag 4: 33,5\% Schmerzreduktion bei anodaler Stimulation vs. 7\% Schmerzzunahme bei Placebo - Stimulation; Tag 5: $35 \%$ Schmerzreduktion bei anodaler Stimulation vs. $1 \%$ Schmerzzunahme bei Placebo - Stimulation; Tag 7: 11\% Schmerzreduktion bei anodaler Stimulation vs. $23 \%$ Schmerzzunahme bei Placebo - Stimulation; Tag 14: 27,5\% Schmerzreduktion bei anodaler Stimulation vs. $5 \%$ Schmerzzunahme bei Placebo - Stimulation; Tag 28: 26,5\% Schmerzreduktion bei anodaler Stimulation vs. $6 \%$ Schmerzzunahme bei Placebo - Stimulation).

Des Weiteren wurden die baseline - VAS - Werte mittels Student - $t$ Test zu allen Zeitpunkten, bei Signifikanz der Zeit in der ANOVA, für die jeweilige Bedingung verglichen. Dabei wies die gesamte Patientengruppe einen signifikanten Unterschied während der anodalen Stimulation (baseline - VAS - Werte verglichen mit VAS - Werten am Tag der 1. Sitzung: $p=0,03 ; 2$. Sitzung: $p=0,03 ; 3$. Sitzung: $p=0,003$, 4. Sitzung: $p=0,001 ; 5$. Sitzung: $p=0,0006$ ) und nach der Stimulation auf (Tag 14: $p=0,03)$, Tag 28: $p=0,03)$. Die höchste Schmerzreduktion wurde nach dem fünften Sitzungstag erreicht (30\% Schmerzreduktion, mittlerer VAS - Wert 4,22 ( $\pm 2,23)$ ). Bei der Placebo - Stimulation ließ sich kein signifikanter Unterschied zwischen den baseline - VAS - Werten und den VAS - Werten während und nach der Stimulation darstellen.

Bei der Analyse der zwölf Patienten aus der Doppelstimulationsgruppe ließ sich eine ähnliche Tendenz verzeichnen: Während der anodalen Stimulation konnte ein signifikanter Unterschied dargestellt werden (baseline - VAS - Werte verglichen mit den VAS - Werten am 1. Tag der Sitzung: $p=0,02 ; 2$. Sitzung: $p=0,05 ; 3$. Sitzung: $p=0,004 ; 4$. Sitzung: $p=0,01 ; 5$. Sitzung: $p=0,008)$. Hierbei konnte die größte Schmerzreduktion mit 37\% bereits am dritten Tag der Sitzung verzeichnet werden (mittlerer VAS - Wert 3,54 ( $\pm 2,29)$ ). In der Placebo - Kontrollgruppe ließ sich bis auf den 1. Sitzungstag $(p=0,05)$ weder für die baseline - VAS - 
Werte noch für die VAS - Werte während und nach der Stimulation eine signifikante Regredienz der Schmerzen eruieren.

Es gab acht Patienten in der anodalen Stimulationsgruppe, die auf die Stimulation ansprachen (63\% der Patienten) $(40 \%$ und mehr Regredienz der VAS - Werte nach 5 Tagen Stimulation) und nur vier Patienten in der Placebo - Gruppe (16\% der Patienten). In der Folgephase nach der Stimulation berichteten vier Patienten aus der anodalen Stimulationsgruppe und drei Patienten aus der Placebo - Stimulationsgruppe von einem langanhaltenden Rückgang der Schmerzen.

Entscheidend ist, dass die Behandlung mit anodalem Gleichstrom bei keinem der Patienten eine Verschlechterung der Schmerzen herbeiführte.

Da nur eine kleine Patientengruppe zur Verfügung stand und die Patientengruppe im Hinblick auf die Schmerzursache heterogen ist, wurden keine Korrelationsanalysen hinsichtlich der Erkrankungsart durchgeführt, jedoch beschrieben Patienten mit Arthrose eine länger andauernde Schmerzreduktion als Patienten mit Rückenschmerzen. In der Gruppe der Fibromyalgie - Patienten konnte weder nach fünf Tagen Behandlung mit Gleichstrom noch im Vergleich zur Placebo - Stimulation eine Schmerzreduktion verzeichnet werden.

\subsection{Nebenwirkungen}

Keiner der Patienten beendete die Stimulation vorzeitig oder brauchte medizinische Interventionen jeglicher Art während oder am Ende der Gleichstromstimulation. In Tabelle 5a sind die beobachteten Nebenwirkungen beider Stimulationsgruppen (anodal und Placebo - Stimulation) während der Stimulationssitzungen dargestellt. Tabelle $5 \mathrm{~b}$ bezieht sich auf die Nebenwirkungen beider Stimulationsgruppen, die die Patienten nach der Stimulation beobachtet haben. 
Tab.5a Nebenwirkungen der tDCS während der Stimulation

\begin{tabular}{|l|c|c|l|r|r|l|l|r|l|}
\hline & \multicolumn{3}{|l|}{ Schmerzen } & \multicolumn{3}{l|}{ Kribbeln } & \multicolumn{2}{l|}{ Jucken } \\
\hline & $\mathrm{N}$ & $\%$ & $\mathrm{Ml}$ & $\mathrm{N}$ & $\%$ & $\mathrm{Ml}$ & $\mathrm{N}$ & $\%$ & $\mathrm{Ml}$ \\
\hline Placebo & 2 & 11,8 & $1 \pm 0$ & 9 & 52,9 & $1,1 \pm 0,11$ & 0 & 0 & 0 \\
\hline Anodal & 2 & 12,5 & $2 \pm 1$ & 10 & 62,5 & $1,3 \pm 0,15$ & 4 & 25 & $1,75 \pm 0,15$ \\
\hline
\end{tabular}

\begin{tabular}{|l|c|c|l|l|l|l|l|l|l|}
\hline & \multicolumn{3}{|l|}{ Brennen } & \multicolumn{3}{l|}{ Müdigkeit } & \multicolumn{3}{l|}{ Nervosität } \\
\hline & $\mathrm{N}$ & $\%$ & $\mathrm{Ml}$ & $\mathrm{N}$ & $\%$ & $\mathrm{Ml}$ & $\mathrm{N}$ & $\%$ & $\mathrm{Ml}$ \\
\hline Placebo & 4 & 23,5 & $1 \pm 0$ & 11 & 64,7 & $1,7 \pm 0,27$ & 1 & 5,9 & $1 \pm 0$ \\
\hline Anodal & 1 & 6,3 & $1 \pm 0$ & 7 & 43,8 & $1,9 \pm 0,46$ & 0 & 0 & 0 \\
\hline
\end{tabular}

\begin{tabular}{|c|c|c|c|c|c|c|c|c|c|}
\hline & \multicolumn{3}{|c|}{$\begin{array}{l}\text { Konzentrations- } \\
\text { störung }\end{array}$} & \multicolumn{3}{|c|}{ Sehstörungen } & \multicolumn{3}{|c|}{ Kopfschmerzen } \\
\hline & $N$ & $\%$ & MI & $\mathrm{N}$ & $\%$ & MI & $\mathrm{N}$ & $\%$ & MI \\
\hline Placebo & 1 & 5,9 & $2 \pm 0$ & 0 & 0 & 0 & 5 & 29,4 & $1,4 \pm 0,24$ \\
\hline Anodal & 1 & 6,3 & $2 \pm 0$ & 2 & 12,5 & $1 \pm 0$ & 4 & 25 & $1,75 \pm 0,75$ \\
\hline
\end{tabular}

\begin{tabular}{|l|c|c|l|}
\hline & \multicolumn{3}{|l|}{ Unangenehm } \\
\hline & $\mathrm{N}$ & $\%$ & $\mathrm{Ml}$ \\
\hline Placebo & 0 & 0 & 0 \\
\hline Anodal & 1 & 6,3 & $3 \pm 0$ \\
\hline
\end{tabular}

\begin{tabular}{|l|l|}
\hline \multicolumn{2}{|l|}{ Sonstige } \\
\hline $\mathbf{N}$ & \\
\hline 1 & Kribbeln in der Zunge \\
\hline 1 & warmes Kribbeln im Bein \\
\hline 1 & Muskelzuckungen \\
\hline
\end{tabular}

Tab.5b Nebenwirkungen der tDCS nach der Stimulation

\begin{tabular}{|l|c|c|l|l|l|l|l|l|l|}
\hline & \multicolumn{3}{|l|}{ Schmerzen } & \multicolumn{4}{l|}{ Kribbeln } & \multicolumn{3}{l|}{ Jucken } \\
\hline & $\mathrm{N}$ & $\%$ & $\mathrm{Ml}$ & $\mathrm{N}$ & $\%$ & $\mathrm{Ml}$ & $\mathrm{N}$ & $\%$ & $\mathrm{Ml}$ \\
\hline Placebo & 1 & 5,9 & $1 \pm 0$ & 1 & 5,9 & $1 \pm 0$ & 1 & 5,9 & $1 \pm 0$ \\
\hline anodal & 1 & 6,3 & $1 \pm 0$ & 1 & 6,3 & $1 \pm 0$ & 1 & 6,3 & $1 \pm 0$ \\
\hline
\end{tabular}




\begin{tabular}{|l|c|c|l|l|l|l|l|l|l|}
\hline & \multicolumn{3}{|l|}{ Brennen } & \multicolumn{3}{l|}{ Müdigkeit } & \multicolumn{3}{l|}{ Nervosität } \\
\hline & $\mathrm{N}$ & $\%$ & $\mathrm{Ml}$ & $\mathrm{N}$ & $\%$ & $\mathrm{Ml}$ & $\mathrm{N}$ & $\%$ & $\mathrm{Ml}$ \\
\hline Placebo & 1 & 5,9 & $1 \pm 0$ & 12 & 70,6 & $2,25 \pm 0,30$ & 1 & 5,9 & $1 \pm 0$ \\
\hline anodal & 1 & 6,3 & $1 \pm 0$ & 5 & 31,3 & $2,5 \pm 0,51$ & 1 & 6,3 & $2 \pm 0$ \\
\hline
\end{tabular}

\begin{tabular}{|l|c|c|l|l|l|l|l|l|r|}
\hline & \multicolumn{4}{|l|}{ Konzentrationsstörung } & \multicolumn{3}{l|}{ Sehstörungen } & \multicolumn{3}{|l|}{ Kopfschmerzen } \\
\hline & $\mathrm{N}$ & $\%$ & $\mathrm{Ml}$ & $\mathrm{N}$ & $\%$ & $\mathrm{Ml}$ & $\mathrm{N}$ & $\%$ & $\mathrm{Ml}$ \\
\hline Placebo & 2 & 11,8 & $2,5 \pm 0,5$ & 0 & 0 & 0 & 6 & 35,3 & $2,2 \pm 0,54$ \\
\hline anodal & 0 & 0 & 0 & 2 & 0 & 0 & 7 & 43,8 & $2 \pm 0,38$ \\
\hline
\end{tabular}

\begin{tabular}{|l|c|c|l|l|l|l|l|l|l|}
\hline & \multicolumn{3}{|l|}{ Übelkeit } & \multicolumn{2}{l|}{ Erbrechen } & \multicolumn{2}{l|}{ Schlafstörungen } \\
\hline & $\mathrm{N}$ & $\%$ & $\mathrm{Ml}(\mathrm{h})$ & $\mathrm{N}$ & $\%$ & $\mathrm{Ml}$ & $\mathrm{N}$ & $\%$ & $\mathrm{Ml}(\mathrm{d})$ \\
\hline Placebo & 1 & 5,9 & $2 \pm 0$ & 0 & 0 & 0 & 2 & 11,8 & $2 \pm 1$ \\
\hline anodal & 0 & 0 & 0 & 0 & 0 & 0 & 4 & 25 & $3 \pm 0,71$ \\
\hline
\end{tabular}

\begin{tabular}{|l|c|c|l|l|l|l|l|l|l|}
\hline & \multicolumn{3}{|l|}{ Manie } & \multicolumn{3}{|l|}{ Kältegefühl } & \multicolumn{3}{l|}{ Wärmegefühl } \\
\hline & $\mathrm{N}$ & $\%$ & $\mathrm{Ml}(\mathrm{h})$ & $\mathrm{N}$ & $\%$ & $\mathrm{Ml}$ & $\mathrm{N}$ & $\%$ & $\mathrm{Ml}$ \\
\hline Placebo & 2 & 11,8 & $3,5 \pm 1,5$ & 1 & 5,9 & $0,25 \pm 0$ & 2 & 11,8 & $1,5 \pm 0,5$ \\
\hline Anodal & 3 & 18,8 & $1,3 \pm 0,33$ & 1 & 6,3 & $1 \pm 0$ & 1 & 6,3 & $2 \pm 0$ \\
\hline
\end{tabular}

\begin{tabular}{|l|l|}
\hline \multicolumn{2}{|l|}{ Sonstige } \\
\hline $\mathrm{N}$ & \\
\hline 1 & Wadenschmerzen \\
\hline 1 & Nacken- / Schulterschmerzen \\
\hline 1 & Steigerung des subjektiven Wohlbefindens \\
\hline
\end{tabular}

Während der Stimulation wurde ein leichtes Kribbeln unter den Elektroden als häufigste Nebenwirkung angegeben (66,6\% der Patienten der anodalen Stimulationsgruppe; 52,9\% der Patienten aus der Placebo Stimulationsgruppe). Die zweithäufigste Nebenwirkung war eine leichte Müdigkeit, die bei $44,4 \%$ in der anodalen und erstaunlicherweise bei $64,7 \%$ in der Placebo - Stimulationsgruppe auftrat.

Auch nach der Stimulation fühlten sich 33,3\% der Patienten der anodalen Stimulations- und 70,6\% der Patienten der Placebo - Stimula- 
tionsgruppe müde. Ebenso traten Kopfschmerzen auf $(38,9 \%$ anodal vs. $35,3 \%$ Placebo).

Über einen Zeitraum von zwei bis fünf Tagen nach der Stimulation klagten vier Patienten über akute Schlafstörungen, in der Placebo - Stimulationsgruppe waren es zwei Patienten.

In der Gruppe der Patienten, die beide Stimulationsarten appliziert bekamen, berichtete nur ein Patient auf explizite Nachfrage, dass er einen Unterschied zwischen beiden Stimulationsarten bemerkt hat.

In der Folgephase nach der Stimulation gab es zwei Patienten, die aus der Studie ausstiegen: Dass sie keine Schmerzreduktion erfahren haben, könnte zu ihrer Entscheidung beigetragen haben (ein Patient aus der anodalen Stimulationsgruppe und ein Patient aus der Placebo Stimulationsgruppe). 


\section{Diskussion}

\subsection{Schmerzreduktion}

Die Ergebnisse dieser Arbeit zeigen, dass die Applikation von anodalem Gleichstrom über dem Hirnareal M1 an fünf aufeinander folgenden Tagen eine Reduktion der Schmerzen bei Patienten mit verschiedenen Schmerztypen erzeugen kann. Hierbei spielt die Reduktion der Schwelle der intrakortikalen Inhibition eine erhebliche Rolle.

Verglichen mit der Studie von Fregni et al (2006a) lag die Schmerzreduktion mittels tDCS bei lediglich $38 \%$ (58\% bei Fregni et al. 2006a). Dies könnte auf die Heterogenität der Patientengruppe zurückzuführen und ein Resultat der unterschiedlichen Kriterien der Schmerzantwort sein. Dennoch sind die Ergebnisse ähnlich zu denen aus Studien mit hochfrequenter rTMS, bei denen eine mittlere Schmerzreduktion von 20-45\% erreicht werden konnte (Lefaucheur et al. 2001; Khedr et al. 2005).

In der hiesigen Studie traten erste Schmerzregredienzen, wenn auch nur mild, bereits unmittelbar nach der ersten Sitzung auf. Eindeutigere Reduktionen ließen sich nach den ersten drei Sitzungen bzw. am vierten und fünften Sitzungstag eruieren. Zu diesem Zeitpunkt divergieren auch die Ergebnisse der Patientengruppe, die die anodale Stimulation erhielten, von denen der Placebo - Gruppe. Bei den Studien von Lefaucheur et al. (2001) zeichnete sich eine optimale Schmerzreduktion nach der ersten Sitzung, welche vier bis fünf Tage nach zusätzlicher rTMS stattfand, ab. Die Frage nach der optimalen Applikationsdauer und Wiederholungsrate wird ein interessanter Aspekt für die Zukunft sein.

Im Vergleich zur Implantation epiduraler Stimulationselektroden sind die Behandlungsergebnisse der tDCS schwächer: So konnte bei der chirurgischen Intervention eine Schmerzreduktion von $28-70 \%$ und eine 
Ansprechrate von 50-80\% - bei jedoch höherem Placeboeffekt - erreicht werden (Tsubokawa et al. 1993; Herregodts et al. 1995; Ebel et al. 1996; Nguyen et al. 1999; Carroll et al. 2000; Nuti et al. 2005). Die hauptsächliche Schmerzreduktion variierte zwischen $28-47 \%$ in der größeren Studienserie (Nguyen et al. 1999; Nuti et al. 2005) und zwischen $50-70 \%$ in der kleineren Studienserien (Herregodts et al. 1995; Ebel et al. 1996). Die MCS mittels implantierter Elektroden scheint trotz des höheren Placeboeffektes und im Vergleich zur fünftägigen Applikation der tDCS einen stärkeren Einfluss auf die Aktivität des Gehirns über mehrere Monate anhaltend zu erreichen.

Die Ergebnisse der längerfristigen Schmerzreduktion mittels anodaler tDCS ähneln denen von Fregni et al. (2006a, 2006b). Genaue Mechanismen, die für die Langzeitwirkung der tDCS verantwortlich sind, konnten bisher nicht eindeutig geklärt werden.

Die Verstärkung der Exzitabilität des M1 - Areals könnte zur Modulation der Schmerzwahrnehmung durch indirekte Effekte in der Schmerzregion, z.B. Thalamus oder subthalamische Kerne, beitragen. Ein Wechsel der Aktivität dieser Kerne ist mittels rTMS (Strafella et al. 2004) und tDCS (Lang et al. 2005) möglich. Eine Aktivierung der thalamischen Kerne kann die Aktivität anderer schmerzassoziierter Strukturen (z. B. anteriore Cinguli, periaquäduktales Grau) zeitweise modifizieren und des Weiteren Schmerzen spinalen Ursprungs inhibieren.

\subsection{Elektrodengröße / Elektrodenposition}

In vielen Studien wurde vermutet, dass die beste Lokalisation für die Schmerzkontrolle mittel rTMS nicht das Areal ist, welches mit der Schmerzrepräsentation korrespondiert, sondern das benachbarte Areal (Lefaucheur et al. 2004). Bezüglich der epidural implantierten Elektroden für die MCS zeigte eine andere Studie, dass die effektivste Position diejenige ist, welche mit der kortikalen somatotopischen Repräsentation der Schmerzwahrnehmung korrespondiert (Nguyen et al. 1999). 
Im Vergleich zu Fregni et al. (2006a, 2006b) wurde in dieser Studie eine kleinere Größe der Stimulationselektroden gewählt, um den Fokus der Stimulation einzugrenzen. Aufgrund der hier gewählten Größe erfolgte die Modulation über einem großen Teil des M1 - Areals, sodass sowohl das M1 - und auch benachbarte Areal aktiviert wurden. Um zu prüfen, ob somatotopisch gestützte Stimulationen mittels tDCS den Analgesie Effekt beeinflussen, sind weitere Studien notwendig.

\subsection{Nebenwirkungen}

Bisherige Untersuchungen zur Frage bezüglich neuronaler Schädigungen oder struktureller Veränderungen des Gehirns nach Applikation von tDCS ergaben keine Hinweise auf schädliche Einflüsse durch die tDCS. So wurde die neuronenspezifische Enolase (NSE) als neuronaler Destruktionsmarker nach tDCS untersucht. Diese war nicht als erhöht zu eruieren. Auch in der kontrastunterstützten MRT sowie mittels EEG konnten keine pathologischen Veränderungen gefunden werden (Nitsche et al .2003a).

Als eine häufige Nebenwirkung ist die wenige Sekunden andauernde Reizung der Kopfhaut, welche von den Probanden als mehr oder weniger schmerzhaftes Kribbeln und Ziehen beschrieben wurde, anzusehen. Kortikale Gewebeschäden wurden bisher nicht nachgewiesen. Jedoch könnte es im Rahmen der Stimulation durch die auf der Kopfhaut aufgelegten Elektroden zu einer chemischen Reaktion und zu Verbrennungen der Kopfhaut kommen. Bei Einhaltung des Sicherheitsprotokolls von Nitsche und Paulus (2000) ist das Risiko einer Hautverbrennung durch Verwendung von in salzwassergetränkten Schwammelektroden jedoch stark minimiert. Mehrere Studien zur Sicherheit der tDCS und zur Evaluation der Nebenwirkungen haben zu klaren Empfehlungen hinsichtlich der sicheren Anwendung geführt. Allen gemein ist, dass diese Methode bei Einhaltung der Richtlinien hinsichtlich gegebener Kontraindikationen und Stimulationsparameter gut verträglich und somit 
nebenwirkungsarm ist (Nitsche et al. 2003b, lyer et al. 2005). Auch in der vorliegenden Arbeit wurden diese Richtlinien eingehalten.

In Bezug auf die Nebenwirkungen, v.a. leichte Müdigkeit und Kribbeln unter den Elektroden, zeigte die Auswertung dieser Arbeit eine höhere Inzidenz dieser als in vorangegangenen Studien mit chronischen Schmerzpatienten (Fregni et al. 2006a, 2006b) und gesunden Probanden (Poreisz et al. 2007). Das größere Ausmaß der Inzidenz der Nebenwirkungen, v.a. Kopfschmerz und Müdigkeit, während und nach der Stimulation im Vergleich zu gesunden Probanden (Poreisz et al. 2007) ist in der vorliegenden Arbeit auf die verschiedenen Grunderkrankungen der Patienten zurückzuführen. So berichteten in vorangegangenen Studien einige Probanden mit einer Migräneerkrankung von häufigerem Auftreten von Nebenwirkungen bei der Behandlung mit tDCS (55,6\% vs. $7,8 \%$ der gesunden Probanden) (Poreisz et al. 2007). Deshalb ist es nicht ungewöhnlich, dass $35-39 \%$ der Patienten Kopfschmerzen verspürten.

Da die Behandlung mit tDCS bisher nur im Rahmen von Studien genutzt wurde, sind die Auswirkungen längerfristiger Stimulationen bezüglich kognitiver und motorischer Funktionen in den verschiedenen Patientengruppen bislang nicht vollständig bekannt. Deshalb können die hier beschriebenen Nebenwirkungen lediglich als vorläufige Sicherheitshinweise angesehen werden.

Nichtsdestotrotz ließen sich auch in dieser Studie keine ernsthaften Nebenwirkungen beobachten.

\subsection{Placebo - Effekt}

Wie bei Furubayashi et al. (2008) berichtet, setzt ab einer Applikationsstärke von 3mA ein Schmerz ein, sodass die Stärke der tDCS mit $1 \mathrm{~mA}$ optimal gewählt wurde. Zudem hat diese niedrige Stärke im Rahmen 
der Placebo - Stimulation eine höhere Chance, vom Patienten unbemerkt zu bleiben, als eine Applikationsstärke von $2 \mathrm{~mA}$.

Außerdem konnte dokumentiert werden, dass der Schweregrad und die therapeutische Refraktärität der Symptome mit den Placeboantworten negativ korrelieren (Rück und Sylvén 2006), sodass die geringe Placeboantwort die Therapierefraktärität unserer Patientengruppe indiziert.

Im Hinblick auf das Auftreten der Nebenwirkungen wie Kribbeln, Brennen und Jucken sowie Kopfschmerzen und Müdigkeit konnten in der Placebo - Gruppe im Vergleich zur Patientengruppe mit anodaler Stimulation höhere Prozentzahlen eruiert werden, sodass davon ausgegangen werden kann, dass die Placebo - Bedingungen optimal gewählt wurden.

\subsection{Klinische Relevanz}

Bei einer nachgewiesenen Schmerzreduktion von bis zu 38\% ist durchaus von einer klinischen Relevanz auszugehen. Verglichen mit der Dauer von Arztbesuchen und anderen Therapieanwendungen (Physiotherapie etc.) ist der Zeitaufwand dieser Methode (20 Minuten täglich an fünf aufeinanderfolgenden Tagen) deutlich geringer. So sind die Kosten der Therapie - abgesehen von den Anschaffungskosten und den Stromkosten - als gering einzustufen. Die Applikation kann nach einem ärztlichen Aufklärungs- und Anamnesegespräch sowie nach einer ersten problemlosen Applikation (unter ärztlicher Aufsicht) im Verlauf auch unter Aufsicht von medizinisch erfahrenem Personal (z.B. medizinisch technische Assistenten, medizinische Fachangestellte) erfolgen. Zudem wäre die Anwendung dieser Methode auch im ambulanten Rahmen (neurologische Praxis, Hausarztpraxis) zu realisieren, sodass die Zahl der Krankenhausaufenthalte und die Aufenthaltsdauer im Krankenhaus deutlich reduziert und somit die Kosten zu Lasten des Gesundheitssystems gesenkt werden können. Auch die Langzeitwirkung der Applikati- 
on bringt Vorteile mit sich: Da die Schmerzreduktion auch über die Applikation hinaus anhielt, ist von einer längeren Arbeitsfähigkeit des $\mathrm{Be}$ troffenen auszugehen. Auch sollte die Frequenz der Arztbesuche regredient sein. Ebenso gesamtwirtschaftlich zu betrachten ist die Reduktion der Kosten für Schmerzmittel.

Bei einem relativ kleinen Nebenwirkungsprofil könnte auch eine Applikation im häuslichen Rahmen in Erwägung gezogen werden. Hierfür sind jedoch zunächst weitere Studien - auch hinsichtlich der Langzeitnebenwirkungen - notwendig.

Aufgrund der bereits langen Dauer der Erkrankung und der damit einhergehenden frustranen Therapieversuche sowie mögliche Persönlichkeitsveränderungen muss zudem mit einer Non - Compliance, welche sich auch in dieser Studie beobachten ließ, des chronischen Schmerzpatienten gerechnet werden.

Weiterhin ist die umfangreiche Anamnese als mögliches Hindernis in Betracht zu ziehen: Dabei ist nicht immer sicher nachzuvollziehen, welche Medikation bereits appliziert wurde und welche weiteren Therapien der Patient bereits erhalten hat. Im Rahmen der Anamneseerhebung sollten auch die Ausschlusskriterien überprüft werden: Das Ausschlusskriterium des Alters, der Schwangerschaft, einer rezeptiven oder globalen Aphasie, einer aktuellen Drogen- / Alkoholabhängigkeit sollten für den Anwender kein Hindernis darstellen bzw. durch wenig Aufwand zu klären sein (Blut- / Urinuntersuchung bezüglich des Vorliegens einer Schwangerschaft oder Nachweis von Alkohol oder Drogen, Aphasie Tests). Auch der Ausschluss einer schwerwiegenden internistischen Erkrankung ist wenig aufwendig, da diese dem Patienten in der Regel bekannt ist oder anhand der aktuellen Medikation (vorausgesetzt diese ist dem Patienten bekannt) auf diese zu schließen ist.

Aufwendiger hingegen ist das Kriterium eines Metallgegenstandes im Körper. So berichten Patienten vom Vorliegen eines implantierten Gegenstandes, sind jedoch nicht immer im Besitz eines 
Implantatausweises, aus welchem hervorgeht, ob es sich um ferromagnetische Materialien handelt. Hier bleibt jedoch die Möglichkeit des Informationsgewinns durch Anforderung der alten Unterlagen (OP - Protokolle). Auch sollte eine psychische Genese der Schmerzen ausgeschlossen sein, z.B. im Rahmen einer Depression mit Hilfe der Hamilton - Rating - Skala für Depressionen oder mittels des Beck - Depressions - Inventars.

Die möglichen und in dieser Studie beobachteten Nebenwirkungen des Kribbelns, der Müdigkeit oder Kopfschmerzen könnten bei der Aufklärung des Patienten zu einer Ablehnung der Applikation führen. Jedoch berichtete ein Großteil der Patienten in dieser Studie, die geringen Nebenwirkungen im Vergleich zu den teilweise jahrelangen Schmerzen in Kauf zu nehmen. Auch hierbei ist der Nutzen gegenüber dem Risiko mit dem Patienten in einem ausführlichen Aufklärungsgespräch abzuwägen. 


\section{Zusammenfassung}

Chronischer Schmerz, dem eine Verletzung des Nervensystems zugrunde liegt, ist assoziiert mit der Dysfunktion verschiedener neuronaler Netzwerke. Die konsekutive Applikation einer nicht invasiven Stimulation, wie die der transkraniellen Gleichstromstimulation, über dem primär motorischen Kortex kann bei der Behandlung von chronischen Schmerzen Anwendung finden, da sie durch Modulation neuronaler Aktivitäten in die neuronalen Netzwerke eingreifen kann. Dies betrifft sowohl das stimulierte als auch die benachbarten Areale. Die vorliegende doppelblinde, randomisierte Studie eruiert den analgetischen Effekt von anodaler und Placebo - Stimulation. Hierfür wurden 23 Patienten mit chronischen und therapierefraktären Schmerzen (Trigeminusneuralgie, Rückenschmerzen, Fibromyalgie, Schmerzsyndrom nach einem Schlaganfall) untersucht. Über eine Dauer von 20 Minuten täglich erhielten sie an fünf aufeinanderfolgenden Tagen eine anodale oder Placebo - Stimulation mit einer Stärke von $1 \mathrm{~mA}$ über dem M1. Der Schmerz wurde einen Monat vor, während und einen Monat nach der Stimulation täglich anhand einer visuellen Analogskala gemessen und in einem Schmerztagebuch dokumentiert. Die Auswertung der ANOVA ergab einen signifikanten Unterschied der Stimulationsarten: So hatten die Placebo- und anodale Stimulation verschiedene Effekte auf Schmerzwahrnehmung. Der Student - $t$ - Test zeigte, dass die anodale Stimulation - auch drei Wochen nach der Applikation - zu einer deutlicheren Besserung der Werte auf der visuellen Analogskala führt. Objektiviert wurden diese Ergebnisse durch die Anwendung von TMS, welche eine Änderung der kortikalen Exzitabilität nachweisen konnte (Antal et al., 2011). Zudem wurden die Nebenwirkungen während und nach der Stimulation erfasst: Es ließen sich keine ernsthaften Nebenwirkungen beobachten, dennoch berichteten sieben Patienten der anodalen Stimu- 
lationsgruppe und sechs Patienten der Placebo - Stimulationsgruppe von Kopfschmerzen.

In Zusammenschau ist die tDCS eine neue, nebenwirkungsarme Methode zur transienten Behandlung von Patienten mit chronischen Schmerzen, die es vermag, Veränderungen des M1 - Areals ähnlich denen, die mittels rTMS und der Implantation von epiduralen Elektroden hervorgerufen werden, zu erzeugen. Obwohl die grundlegenden neuronalen Mechanismen aller Methoden (rTMS, epidurale Elektroden, tDCS) unterschiedlich zu sein scheinen, vermögen sie dennoch eine Änderung der Aktivität verbundener Areale des Gehirns herbeizuführen und somit Einfluss auf die Schmerzwahrnehmung zu nehmen. 


\section{Literaturverzeichnis}

Antal A, Nitsche M (2004): Facilitation of visuo - motor learning by transcranial direct current stimulation of the motor and extrastriate visual areas in humans. Eur J Neurosci 19: 2888-2892

Antal A, Nitsche M, Paulus W (2008): Transcranial direct current stimulation and the visual cortex. Brain Res Bull 68: 459-463

Antal A, Paulus W, Nitsche M (2011): Electrical stimulation and visual network plasticity. Restor Neurol Neurosci 29: 365-374

Backonja M, Betdoun A, Edwards KR, Schwarz SL, Fonseca V, Hes M, LaMoreaux L, Garofalo E (1998): Gabapentin for the symptomatic treatment of painful neuropathy in patients with diabetes mellitus. JAMA 280: $1831-1836$

Bikson M, Inoue M, Akiyama H, Deans J, Fox J, Miyakawa H, Jefferys J (2004): Effects of uniform extracellular DC electric fields on excitability in rat hippocampal slices in vitro. J Physiol 557: 175-190

Bindman LJ, Lippold OCJ, Redfearn JWT (1964): The action of brief polarizing currents on the cerebral cortex of the rat (1) during current flow and (2) in the production of long - lasting after - effects. J Physiol 172: $369-382$

Boggio P, Aparecida Valasek C, Campanha C, Alem Giglio A, Baptista N, Morgan Lapenta O , Fregni F (2011): Non - invasive brain stimulation 
to assess and modulate neuroplasticty in Alzheimer's disease. Neuropsychol Rehabil 21: 703-716

Bolton JE, Wilkinson RC (1998): Responsiveness of pain scales: a comparison of three pain intensity measures in chiropractic patients. $J$ Manipulative Physiol Ther 21: 1-7

Boros K, Poreisz C, Münchau A, Paulus W , Nitsche MA (2008): Premotor transcranial direct current stimulation (tDCS) affects primary motor excitability in humans. Eur J Neurosci 27: 1292-1300

Carroll D, Joint C, Maartens N, Shlugman D, Stein J, Aziz TZ (2000): Motor cortex stimulation for chronic neuropathic pain: a preliminary study of 10 cases. Pain $\underline{84}$ : 431-437

Chaieb L, Antal A, Paulus W (2008): Gender - spezific modulation of short - term neuroplasticity in the visual cortex induced by transcranial direct current stimulation. Visual Neuroscience 25: 77-81

Creutzfeldt OD, From GH, Kapp H (1962): Influence of transcortical DC currents on cortical neuronal activity. Exp Neurol $\underline{5}$ : 436-452

Dasilva AF, Mendonca ME, Zaghi S, Lopes M, Dossantos MF, Spierings EL, Bajwa Z, Datta A, Bikson M, Fregni F (2012): tDCS - induced analgesia and electrical fields in pain - related neural networks in chronic migraine. Headache, article first published online, 18 Apr 2012, doi: 10.1111/j.1526 - 4610.2012.02141.x (letzter Zugriff 22.05.2012) 
Donoghue JP (1995): Plasticity of adult sensorimotor representations. Curr Opin Neurobiol $\underline{5}$ : 749-754

Dworkin RH, Corbin AE, Young JP, Sharma U, LaMoreaux L, Garofalo EA, Poole RM (2003): Pregabalin for the treatment of postherpetic neuralgia: a randomized, placebo - controlled trial. Neurology 22: 12741283

Ebel H, Rust D, Tronnier V, Böker D, Kunze S (1996): Chronic precentral stimulation in trigeminal neuropathic pain. Acta Neurochir 138: 1300-1306

Eisenberg E, Lurie Y, Braker C, Daoud D, Ishay A (2001): Lamotrigine reduces painful diabetic neuropathy. A randomized and controlled study. Neurology 57: 505-509

Endres HG, Victor N, Haake M, Witte S, Streitberger K, Zenz M (2007): Acupuncture for the Treatment of Chronic Knee and Back Pain. Dtsch. Ärztebl 104 (3): 123-130

Fenton BW, Palmieri PA, Boggio P, Fanning J, Fregni F (2009): A preliminary study of transcranial direct current stimulation for the treatment of refractory chronic pelvic pain. Brain Stimul 2: 103-107

Fregni F, Boggio P, Nitsche M, Marcolin M, Rigonatti S, Pascual Leone A (2006a): Treatment of major depression with transcranial direct current stimulation. Bipolar Disord $\underline{8}$ : 203-205 
Fregni F, Boggio P, Nitsche M, Rigonatti S, Pascual - Leone A (2006b): Cognitive effects of repeated sessions of transcranial direct current stimulation in patients with depression. Depress Anxiety 23: 1-3

Fregni F, Boggio PS, Lima MC, Ferreira MJL, Wagner T, Rigonatti SP, Castro AW, Souza DR, Riberto M, Freedman S et al. (2006c): A sham controlled, phase II trial of transcranial direct current stimulation for the treatment of central pain in traumatic spinal cord injury. Pain 122: 197209

Fregni F, Gimenes R, Valle AC, Ferreira MJL, Rocha RR, Natalle L, Bravo R, Rigonatti SP, Freedman SD, Nitsche MA et al. (2006d): A randomized sham - controlled, proof of principle study of transcranial direct current stimulation for the treatment of pain in fibromyalgia. Arthritis Rheum 54: 3988-3998

Furubayashi T, Terao Y, Arai N, Okabe S, Mochizuki H, Hanajima R, Hamada M, Yugeta A, Inomata - Terada S, Ugawa Y (2008): Short and long duration transcranial direct current stimulation (tDCS) over the human hand motor area. Exp Brain Res 185: 279-286

Herregodts P, Stadnik T, De Ridder F, D`Heans J (1995): Cortical stimulation for central neuropathic pain: 3 - D surface MRI for easy determination of the motor cortex. Acta Neurochir Suppl 64: 132-135

http://de.academic.ru/pictures/dewiki/103/gray756.png (letzter Zugriff 16.12.2011) 
http://nwg.glia.mdc-berlin.de/en/courses/method/2010/01.html (letzter Zugriff 16.12.2011)

http://www.iasp-pain.org/AM/Template.cfm?Section=Pain_Defi...isplay. cfm\&ContentID=1728 (letzter Zugriff 16.12.2011)

http://www.neuroconn.de/de/loesungen/dc-stimulator/ (letzter Zugriff 16.12.2011)

lyer M, Mattu U, Grafman J, Lomarev M, Sato S, Wassermann E (2005): Safety and cognitive effect of frontal DC brain polarization in healthy individuals. Neurology $\underline{64}: 872-875$

Kahle W in: Taschenatlas der Anatomie - in 3 Bänden, Band III: Nervensystem und Sinnesorgane; hrsg. von Werner Kahle fortgeführt von Michael Frotscher, Thieme - Verlag Stuttgart, 2002, 308, 312, 324

Khedr EM, Kotb H, Kamel NF, Ahmed MA, Sadek R, Rothwell JC (2005): Longlasting analgetic effects of daily sessions of repetitive transcranial magnetic stimulation in central and peripheral neuropathic pain. J Neurol Neurosurg Psychiatr $\underline{76}$ : 833-838

Kincses T, Antal A (2004): Facilitation of probabilistic classification learning by transcranial direct current stimulation of the prefrontal cortex in the human. Neuropsychologia $\underline{42}:$ 113-117

Kröner - Herwig B: Biofeedback; in: Psychologische Schmerztherapie Grundlagen, Diagnostik, Krankheitsbilder, Behandlung, hrsg. von 
Heinz-Dieter Basler, Carmen Franz, Beate Kröner Herwig, Hans Peter Rehfisch, Hanne Seemann, Springer Verlag Heidelberg, 1999, 627-644

Kujirai T, Caramia MD, Rothwell JC, Day BL, Thompson PD, Ferbert A, Wroe S, Asselma P, Marsden CD (1993): Corticocortical inhibition in human motor cortex. J Physiol 471: 501-519

Lang N, Siebner HR, Ward NS, Lee L, Nitsche MA, Paulus W, Rothwell JC, Lemon RN, Frackowiak RS (2005): How does transcranial DC stimulation of the primary motor cortex alter regional neuronal activity in the human brain?. Eur J Neurosci 22: 495-504

Lefaucheur JP, Drouot X, Keravel Y, Nguyen JP (2001): Pain relief induced by repetitive transcranial magnetic stimulation of precentral cortex. Neuroreport 12: 2963-2965

Lefaucheur JP, Drouot X, Menard - Lefaucheur I, Zerah F, Bendib B, Cesaro P, Keravel Y, Ngyuen JP (2004): Neurogenic pain relief by repetitive transcranial magnetic cortical stimulation depends on the origin and the site of pain. J Neurol Neurosurg Psychiatr $\underline{75}$ : 612-616

Liebetanz D, Nitsche MA, Tergau F, Paulus W (2002): Pharmacological approach to the mechanism of transcranial DC - stimulation - induced after - effects of human motor cortex excitability. Brain 125: 2238-2247

Max MB, Culnane M, Schafer SC, Gracely RH, Walther DJ, Smoller B, Dubner R (1987): Amitriptyline relives diabetic neuropathy pain in patients with normal and depressed mood. Neurology 37: 589-596 
Max MB, Lynch SA, Muir J, Shoaf SE, Smoller B, Dubner R (1992): Effects of desipramine, amitriptyline and fluoxetine on pain in diabetic neuropathy. N Engl J Med 325: 1250-1256

McQuay HJ, Carroll D, Jadad AR, Wiffen P, Moore A (1995): Anticonvulsant drugs for management of pain: a systematic review. BMJ $\underline{311}$ : 1047-1052

McQuay HJ, Tramer M, Nye BA, Carroll D, Wiffen PJ, Moore RA (1996): A systematic review of antidepressants in neuropathic pain. Pain 68: 217-227

Meyerson BA, Lindblom U, Linderoth B, Lind G, Herregodts P (1993): Motor cortex stimulation as treatment of trigeminal neuropathic pain. Acta Neurochir Suppl 58: 150-153

Mori $\mathrm{F}$, Codecà $\mathrm{C}$, Kusayanagi $\mathrm{H}$, Montelone $\mathrm{F}$, Buttari $\mathrm{F}$, Fiore $\mathrm{S}$, Bernardi G, Koch G, Centonze D (2010): Effects of anodal transcranial dirrect current stimulation on chronic neuropathic pain in patients with multiple sclerosis. J Pain 11: 436-442

Morley S, Eccleston C, Williams A (1999): Systematic review and meta - analysis of randomized controlled trials of cognitive behaviour therapy and behaviour therapy for chronic pain in adults, excluding headache. Pain 80: 1-13 
Nguyen JP, Lefaucheru JP, Decq P, Uchiyama T, Carpentier A, Fontaine $D$, Brugières $P$, Pollin $B$, Fève $A$, Rostaing $S$ et al. (1999): Chronic motor cortex stimulation in the treatment of central and neuropathic pain. Correlations between clinical, electrophysiological and anatomical data. Pain $\underline{82}$ : 245-251

Nitsche MA, Paulus W (2000): Excitability changes induced in the human motor cortex by weak transcranial direct current stimulation. $\mathrm{J}$ Physiol 527: 633-639

Nitsche MA, Paulus W (2001): Sustained excitability elevations induced by transcranial DC motor cortex stimulations in humans. Neurology $\underline{57}$ : 1899-1901

Nitsche MA, Liebetanz D, Antal A, Lang N, Tergau F, Paulus W (2003a): Modulation of cortical excitability by weak direct current stimulation - technical, safety and functional aspects. Suppl Clin Neurophysiol 56: 255-276

Nitsche MA, Liebetanz D, Lang N, Antal A, Tergau F, Paulus W (2003b): Safety criteria for transcranial direct current stimulation (tDCS) in humans. Clin Neurophysiol 114: 2220-2222

Nitsche MA, Cohen LG, Wassermann E, Priori A, Lang N, Antal A, Paulus W, Hummel F, Boggio P, Fregni F, Pascual - Leone A (2008): Transcranial direct current stimulation: State of the art 2008. Brain Stimul 1: 206-223 
Nitsche MA, Kuo MF, Karrasch R, Wächter B, Liebetanz D, Paulus W (2010): Serotonin affects tDCS - induced neuroplasticity in humans. Biol Psychiatry 65: 503-508

Nix WA (1998): Was ist gesichert in der Schmerztherapie? Haben Neuroleptika eine analgetische Potenz? Eine Metaanalyse. Schmerz 12: 30-38

Nuti C, Peyron R, Garcia - Larrea L, Brunon J, Laurent B, Sindou M, Mertens $P$ (2005): Motor cortex stimulation for refractory neuropathic pain: Four year outcome and predictors of efficacy. Pain 118: 43-52

Onghena P, Van Houdenhove B (1992): Antidepressant - induced in chronic non - malignant pain: a meta - analysis of 39 placebo - controlled studies. Pain $\underline{49}: 205-219$

Poreisz C, Boros K, Antal A, Paulus W (2007): Safety aspects of transcranial direct current stimulation concerning healthy subjects and patients. Brain Res Bull 72: 208-214

Rehfisch HP, Basler HD: Entspannung und Imagination; in: Psychologische Schmerztherapie - Grundlagen, Diagnostik, Krankheitsbilder, Behandlung, hrsg. von Heinz-Dieter Basler, Carmen Franz, Beate Kröner Herwig, Hans Peter Rehfisch, Hanne Seemann, Springer Verlag Heidelberg, 1999, 601-626

Riberto M, Marcon Alfieri F, Monteiro de Benedetto Pacheco K, Dini Leite V, Nemoto Kaihami H, fregni F, Rizzo Battistella L (2011): Efficacy of transcranial direct current stimulation coupled with a mutlidisciplinary 
rehabilitation program for the treatment of fibromyalgia. Open Rheumatol J $\underline{5}: 45-50$

Rowbotham M, Harden N, Stacey B, Bernstein P, Magnus - Miller L (1998): Gabapentin for the treatment of postherpetic neuralgia. JAMA 280: 1837-1842

Rollnik JD, Wüstefeld S, Däuper J, Karst M, Fink M, Kossev A, Dengler $R$ (2002): Repetitive transcranial magnetic stimulation for the treatment of chronic pain - a pilot study. Eur Neurol 4ㅇ: 6-10

Rück A, Sylvén C (2006): "Improvement" in the placebo group could be due to regression to the mean as well as to sociobiologic factors. Am J Cardiol 97: 152-153

Rush S, Driscoll DA (1968): Current distribution in the brain from surface electrodes. Anesth Analg 47: 717-723

Saag KG, Criswell LA, Sems KM, Nettleman MD, Kolluri S (1996): Low dose corticosteroids in rheumatoid arthritis: A meta - analysis of their moderate - term effectiveness. Arthritis Rheum 39: 1818-1825

Schäfer M, Stein C (1997): Schmerzen in der postoperativen Phase medizinische und ökonomische Aspekte. Anaesthesist 46: 120-123

Siebner $\mathrm{H}$, Ziemann U: Hirnstimulation - physiologische Grundlagen; in: Das TMS - Buch, hrsg. von Hartwig Roman Siebner und Ulf Ziemann, Springer Verlag Berlin, 2007, 28-45 
Smith LA, Oldham AD, McQuay AJ, Moore RA (2008): Teasing apart quality and validity in systematic reviews: an example from acupuncture trials in chronic neck and back pain. Pain $\underline{86}$ : 119-132

Steinbach JP, Diener HC: Akuter und chronischer Schmerz; in: Therapie und Verlauf neurologischer Erkrankungen; hrsg. von Thomas Brandt, Johannes Dichgans, Hans-Christoph Diener; Kohlhammer Verlag Stuttgart, 2007, 104-118

Strafella AP, Vanderwerf Y, Sadikot AF (2004): Transcranial magnetic stimulation of the human motor cortex influences the neuronal activity of subthalamic nucleus. J Neurosci 20: 2245-2249

Thirugnanasambandam N, Sparing R, Dafokatis M, Meister IG, Paulus W, Nitsche MA, Fink GR (2011): Isometric contraction interferes with transcranial direct current stimulation (tDCS) induced Plasticity: evidence of state - dependent neuromodulation in human motor cortex. Restor Neurol Neurosci 29: 311-320

Tsubokawa T, Katyama $\mathrm{Y}$, Yamamoto $\mathrm{T}$, Hirayama T, Koyama $\mathrm{S}$ (1993): Chronic motor cortex stimulation in patients with thalamic pain. J Neurosurg 78: 393-401

Valle A, Roizenblatt S, Botte S, Zaghi S, Riberto M, Tufik S, Boggio P, Fregni $F$ (2009): Efficacy of anodal transcranial direct current stimulation (tDCS) for the treatment of fibromyalgia: results of a randomized, sham - controlled long clinical trial. J Pain 2: 353-361 
Valls - Sole J, Pascual - Leone A, Wassermann EM, Hallet M (1992): Human motor evoked responses to paired transcranial magnetic stimuli. Electorencephalogr Clin Neurphysiol 85: 355-364

van Tulder MW, Cherkin DC, Berman B, Lao L, Koes BW: Acupuncture for low back pain (Cochrane Review). The Cochrane Library, Issue 1, Oxford: Update Software 2001

Venkatakrishnan A, Contreras - Vidal JL, Sandrini M, Cohen LG (2011): Independent component analysis of resting brain activity reveals transient modulation of local cortical processing by transcranial direct curreent stimulation. IEEE Eng Biol Soc: 8102-8105

Vestergaard K, Andersen G, Gottrup H, Kristensen BT, Jensen TS (2001): Lamotrigine for central poststroke pain. A randomized controlled trial. Neurology $\underline{56}$ : 184-190

Wallace BA, Ashkan K, Benabid AL (2004): Deep brain stimulation for the treatment of chronic, intractable pain. Neurosurg Clin N Am 15: 343357

Wiffen P, Collons S, McQuay H, Carroll D, Jadad A, Moore A: Anticonvulsant drugs for acute and chronic pain (Cochrane Review). The Cochrane Library Issue 4, Oxford: Update Software 2001

World Medical Organization (1996): Declaration of Helsinki. BMJ $\underline{313}$ : 1448-1449 


\section{Anhang}

\subsection{Aufklärungsbogen zur Teilnahme an der Studie Georg-August-Universität Göttingen • Stiftung Öffentlichen Rechts Be- reich Humanmedizin • Universitätsklinikum • Medizinische Fakultät}

Zentrum Neurologische Medizin

Abteilung Neurologie (Prof. Dr. M. Bähr)

Abteilung Klinische Neurophysiologie (Prof. Dr. W. Paulus)

\section{Telefon: 0551-39-6650/6652}

\section{Information für Teilnehmerinnen und Teilnehmer über die Untersuchung:}

Überprüfung der Effektivität schwacher transkranieller Gleichstromstimulation bei Patienten mit Chronischen Schmerzen

Sehr geehrte Teilnehmerin, sehr geehrter Teilnehmer!

Wir bedanken uns für Ihr Interesse an der o.g. Studie und möchten Ihnen den Ablauf der Studie anhand dieses Informationsbogens erläutern. Sie selbst werden nicht unmittelbar von dieser Studie profitieren, die Ergebnisse ermöglichen es uns jedoch, weiterführende Erkenntnisse über die Ursachen und Therapiemöglichkeiten Ihrer Erkrankung zu gewinnen.

Es soll überprüft werden, ob sich die Symptomatik chronischer Schmerzen durch die Applikation schwachen Gleichstroms über die Kopfhaut verändert. Es wird angenommen, dass bei Ihrer Erkrankung ein Ungleichgewicht in der Aktivität der Hirnrinde vorliegt. Bei gesunden Probanden ist transkranielle (=durch den Schädelknochen) Gleichstromstimulation in der Lage, die Erregbarkeit der Hirnrinde zu verändern. Es soll nun überprüft werden, ob diese Maßnahme bei Schmerzpatienten wirksam ist.

Die Untersuchung dauert insgesamt etwa 7 Stunden, verteilt auf 7 Termine. In der ersten Sitzung sollen Sie jeweils Fragen zu Ihren Symptomen beantworten, die Ihnen auf Fragebögen gestellt werden. In den weiteren Sitzungen werden zwei Elektroden 
auf Ihrer Kopfhaut angebracht. Über diese Elektroden fließt während der Untersuchung für 20 Minuten ein schwacher Gleichstrom. Dieser Strom ist für Sie nicht oder allenfalls sehr geringfügig wahrnehmbar. Dieses nicht invasive (=nicht verletzende) und schmerzfreie Verfahren wurde bereits in mehreren Untersuchungen angewendet und hat sich als risiko- und nebenwirkungsarm erwiesen. Selten und lediglich nach kontinuierlicher Gleichstromapplikation über Stunden traten leichte Kopfschmerzen und Hautreizungen im Bereich der Elektroden auf, die sich relativ schnell vollständig zurückbildeten. Sie werden eine wirksame oder eine nicht wirksame Stimulation erhalten, eine so genannte Placebo-Stimulation. Um Erwartungseffekte zu vermeiden, wird Innen die Art der Stimulation nicht mitgeteilt. Vor und nach der Stimulation bitten wir Sie, einen Monat lang ein Schmerztagebuch zu führen.

Selbstverständlich ist es Ihnen jederzeit möglich, ohne Angabe von Gründen und ohne dass Ihnen hieraus Nachteile entstehen, von der Teilnahme an der Studie zurückzutreten. Nachhaltige Wirkungen werden durch diese Untersuchung nicht angestrebt und sind auch nicht zu erwarten.

Falls einer der folgenden Punkte auf Sie zutrifft, ist eine Teilnahme an der Studie nicht möglich:

1. Andere neurologische Erkrankungen, akut oder in der Vorgeschichte

2. Alter $<18$ oder $>70$.

3. Metallimplantate im Kopf oder Herzschrittmacher, ferromagnetische Objekte / andere Objekte mit Risiken für den Kontakt mit den Elektroden.

4. Schwangerschaft oder Stillperiode.

5. Vorliegen einer gravierenden internistischen (Erkrankung der inneren Organe) oder psychiatrischen (seelische Erkrankung) Vorerkrankung, insbesondere Schizophrenie (seelische Erkrankung mit Wahn, Trugwahrnehmungen und Störungen des Denkens) oder Manie (Erkrankung des Gemütes mit gehobener Stimmung).

6. Drogen-, Medikamenten- und/oder Alkoholabhängigkeit

7. Rezeptive oder globale Aphasie (Störung des Sprachverständnisses bzw. zusätzlich des Sprechens)

8. Teilnahme an einer anderen klinischen Studie innerhalb von 8 Wochen vor Studieneinschluss. 
Die aus der Studie gewonnenen Daten werden pseudonymisiert wissenschaftlich ausgewertet. Ein Rückgriff auf personenbezogene Daten bei Widerruf der Studienteilnahme ist möglich. Alle Erfordernisse des Datenschutzes werden dabei beachtet. Wir weisen darauf hin, dass pseudonymisierte Daten im Rahmen der Dokumentations- und Mitteilungspflichten nach $\S 12$ und $\S 13$ der Verordnung über die Anwendung der Guten Klinischen Praxis bei der Durchführung von klinischen Prüfungen mit Arzneimitteln zur Anwendung am Menschen (GCP-Verordnung) weitergeleitet werden können; betroffene Personen, die der Weitergabe nicht zustimmen, können nicht in die klinische Prüfung eingeschlossen werden. Die Teilnahme an der Studie ist freiwillig, es besteht das Recht auf Nicht-Teilnahme. Sie haben jederzeit die Möglichkeit, ohne Angabe von Gründen und ohne Nachteile für weitere Behandlungen die Teilnahme zu widerrufen.

PD. rer. nat. Andrea Antal

PD Dr. med. Martin Sommer

Abteilung Klinische Neurophysiologie

Abteilung Klinische Neurophysiologie

Prof. Dr. med. Walter Paulus

Leiter der Abteilung

Abteilung Klinische Neurophysiologie 
Georg-August-Universität Göttingen • Stiftung Öffentlichen Rechts Bereich Humanmedizin • Universitätsklinikum • Medizinische Fakultät

Zentrum Neurologische Medizin

Abteilung Neurologie (Prof. Dr. M. Bähr)

Abteilung Klinische Neurophysiologie (Prof. Dr. W. Paulus)

\section{Telefon: 0551-39-6650/6652}

\section{EINVERSTÄNDNISERKLÄRUNG ZUR TEILNAHME AN DER UNTERSU- CHUNG:}

Überprüfung der Effektivität schwacher transkranieller Gleichstromstimulation bei Patienten mit Chronischen Schmerzen

Herr/Frau Dr. hat mir heute anhand der Hinweise auf dem Informationsbogen für Teilnehmer an o.g. Studie die Durchführung der Untersuchung erläutert.

Ich habe diesbezüglich keine weiteren Fragen mehr und willige hiermit in die dargestellten Untersuchungen ein. Alle mich interessierenden Fragen wurden ausreichend beantwortet.

Ich erkläre darüber hinaus, dass ich alle Angaben zur Krankengeschichte wahrheitsgemäß gemacht habe.

Mir ist bekannt, dass ich jederzeit ohne Angabe von Gründen die weitere Untersuchung ablehnen bzw. meine Einverständniserklärung widerrufen kann, ohne dass mir daraus Nachteile entstehen.

Ich bin bereit, an der Studie teilzunehmen.

Ich bin damit einverstanden, dass Daten, welche sich aus der Studie ergeben, ohne Erwähnung meiner Identität anonym veröffentlicht werden. Alle Befunde sind vertraulich und werden unter strikter Einhaltung des Datenschutzes gehandhabt.

Eine Kopie dieser Erklärung wurde mir überreicht.

Göttingen, den 
Name des aufklärenden Arztes (in Druckbuchstaben) und Unterschrift

Name des Probanden (in Druckbuchstaben) und Unterschrift 


\subsection{Schmerztagebuch / Schmerzskala}

Abb. 6 Visuelle Analogskala
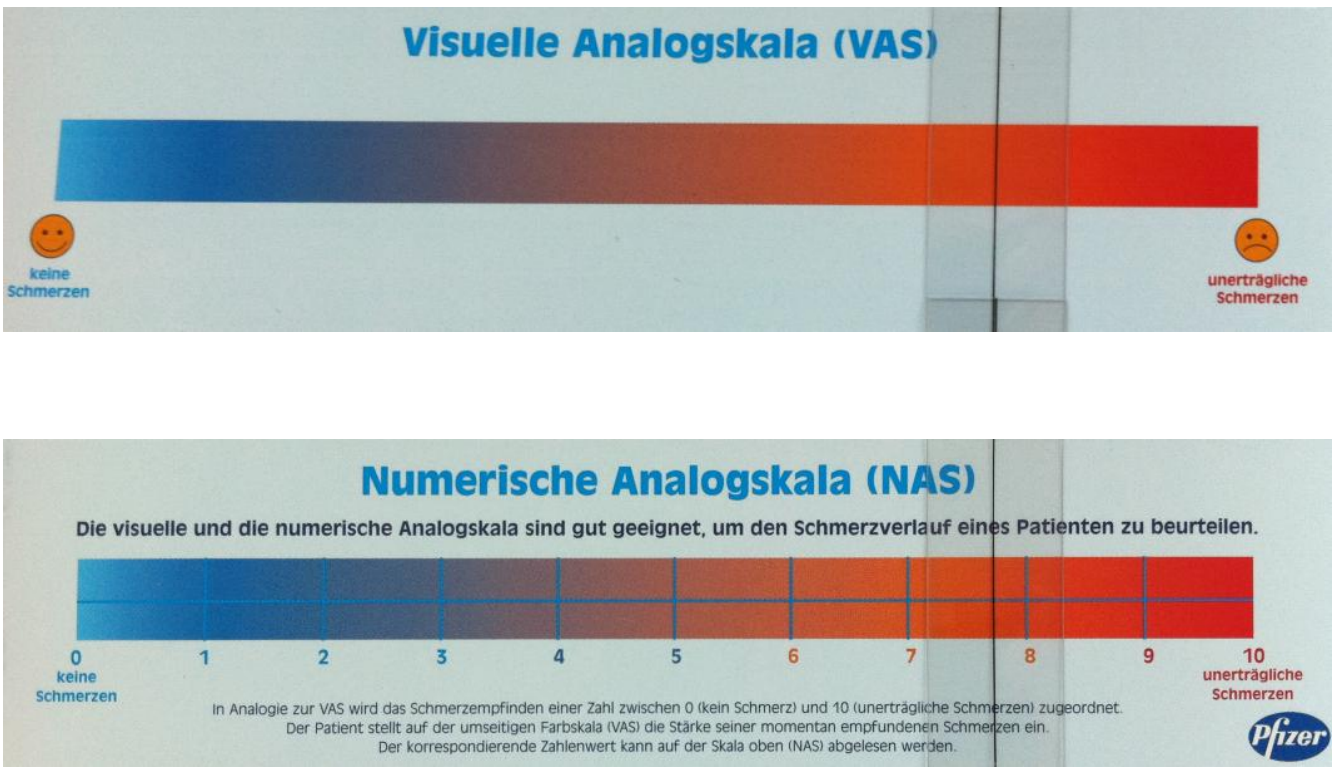

Abb. 7 Auszug Schmerztagebuch ( $F=F r u ̈ h ; M=M i t t a g, ~ A=A b e n d)$

\begin{tabular}{|c|c|c|c|c|c|c|c|c|c|c|c|c|c|c|c|c|c|c|c|c|}
\hline \multicolumn{3}{|c|}{ Mo } & \multicolumn{3}{|c|}{$\mathbf{D i}$} & \multicolumn{3}{|c|}{ Mi } & \multicolumn{3}{|c|}{ Do } & \multicolumn{3}{|c|}{$\mathrm{Fr}$} & \multicolumn{3}{|c|}{$\mathrm{Sa}$} & \multicolumn{3}{|c|}{ So } \\
\hline$F$ & $\mathbf{M}$ & $\mathbf{A}$ & $F$ & $\mathbf{M}$ & $\mathbf{A}$ & $\bar{F}$ & M & $\mathbf{A}$ & $\bar{F}$ & M & $\mathbf{A}$ & $F$ & $\mathbf{M}$ & A & $F$ & $\mathbf{M}$ & $\mathbf{A}$ & $F$ & $\mathbf{M}$ & $\mathbf{A}$ \\
\hline 10 & 10 & 10 & 10 & 10 & 10 & 10 & 10 & 10 & 10 & 10 & 10 & 10 & 10 & 10 & 10 & 10 & 10 & 10 & 10 & 10 \\
\hline 9 & 9 & 9 & 9 & 9 & 9 & 9 & 9 & 9 & 9 & 9 & 9 & 9 & 9 & 9 & 9 & 9 & 9 & 9 & 9 & 9 \\
\hline 8 & 8 & 8 & 8 & 8 & 8 & 8 & 8 & 8 & 8 & 8 & 8 & 8 & 8 & 8 & 8 & 8 & 8 & 8 & 8 & 8 \\
\hline 7 & 7 & 7 & 7 & 7 & 7 & 7 & 7 & 7 & 7 & 7 & 7 & 7 & 7 & 7 & 7 & 7 & 7 & 7 & 7 & 7 \\
\hline 6 & 6 & 6 & 6 & 6 & 6 & 6 & 6 & 6 & 6 & 6 & 6 & 6 & 6 & 6 & 6 & 6 & 6 & 6 & 6 & 6 \\
\hline 5 & 5 & 5 & 5 & 5 & 5 & 5 & 5 & 5 & 5 & 5 & 5 & 5 & 5 & 5 & 5 & 5 & 5 & 5 & 5 & 5 \\
\hline 4 & 4 & 4 & 4 & 4 & 4 & 4 & 4 & 4 & 4 & 4 & 4 & 4 & 4 & 4 & 4 & 4 & 4 & 4 & 4 & 4 \\
\hline 3 & 3 & 3 & 3 & 3 & 3 & 3 & 3 & 3 & 3 & 3 & 3 & 3 & 3 & 3 & 3 & 3 & 3 & 3 & 3 & 3 \\
\hline 2 & 2 & 2 & 2 & 2 & 2 & 2 & 2 & 2 & 2 & 2 & 2 & 2 & 2 & 2 & 2 & 2 & 2 & 2 & 2 & 2 \\
\hline 1 & 1 & 1 & 1 & 1 & 1 & 1 & 1 & 1 & 1 & 1 & 1 & 1 & 1 & 1 & 1 & 1 & 1 & 1 & 1 & 1 \\
\hline 0 & 0 & 0 & 0 & 0 & 0 & 0 & 0 & 0 & 0 & 0 & 0 & 0 & 0 & 0 & 0 & 0 & 0 & 0 & 0 & 0 \\
\hline
\end{tabular}




\subsection{Fragebogen zur Erfassung von Nebenwirkungen}

\section{Fragebogen über die Gleichstromstimulation}

1. Geschlecht:

Männlich

Weiblich

2. Alter:

3. In wie vielen Untersuchungen haben Sie teilgenommen?

4. Haben Sie diese Untersuchungen als gesunde/r Proband/in oder als Patient/in $\square$ mitgemacht?

Wegen welcher Krankheit haben Sie eine Untersuchung mit der Gleichstromstimulation mitgemacht?

\section{5. Über welcher Region wurden Sie stimuliert?}

$\square$ motorische Hirnrinde (eine Elektrode über dem li. Schädel und die andere über der re. Augenbraue oder umgekehrt)

Sehrinde (eine Elektrode am Hinterkopf und die andere über der Mitte des Schädels)

parietale Hirnrinde (eine Elektrode hinter dem Ohr und die andere über der Mitte des Schädels)

$\square$ frontale Hirnrinde (eine Elektrode über dem li. vorderen Schädel und die andere über der re. Augenbraue oder umgekehrt) 
6. Haben Sie bei Beginn und/oder Ende der Stimulation einen Lichtblitz wahrgenommen?

\begin{tabular}{|l|l|}
\hline$\square$ ja & $\square$ nein \\
\hline
\end{tabular}

7. Haben Sie während der Stimulation unter den Elektroden Schmerz gefühlt?

\begin{tabular}{|l|l|}
\hline$\square$ ja & $\square$ nein \\
\hline
\end{tabular}

Wenn ja, wie stark?

$\square$-gering $\square$-mäßig $\square$-mittelmäßig $\square$-stark $\square$-nicht aushaltbar

8. Hat ihre Kopfhaut während der Stimulation unter den Elektroden gekribbelt?

\begin{tabular}{|l|l|}
\hline$\square$ ja & $\square$ nein \\
\hline
\end{tabular}

Wenn ja, wie stark?

$\square$-gering $\square$-mäßig $\square$-mittelmäßig $\square$-stark $\square$-nicht aushaltbar

9. Hat ihre Kopfhaut während der Stimulation unter den Elektroden gejuckt?

\begin{tabular}{|l|l}
\hline$\square$ ja & $\square$ nein \\
\hline
\end{tabular}

Wenn ja, wie stark?

$\square$-gering $\square$-mäßig $\square$-mittelmäßig $\square$-stark $\square \quad$-nicht aushaltbar 
10. Hat ihre Kopfhaut während der Stimulation unter den Elektroden gebrannt?

\begin{tabular}{|l|l|}
\hline$\square$ ja & $\square$ nein \\
\hline
\end{tabular}

Wenn ja, wie stark?

$\square$-gering $\square$-mäßig $\square$-mittelmäßig $\square$-stark $\square$-nicht aushaltbar

11. Waren Sie während der Stimulation müde?

\begin{tabular}{|l|l|}
\hline$\square$ ja & $\square$ nein \\
\hline
\end{tabular}

Wenn ja, wie müde waren Sie?

$\square$-gering $\square$-mäßig $\square$-mittelmäßig $\square$-stark $\square$-äußert

12. Waren Sie während der Stimulation nervös?

\begin{tabular}{|l|l|}
\hline$\square$ ja & $\square$ nein \\
\hline
\end{tabular}

Wenn ja, wie nervös waren Sie?

$\square$-gering $\square$-mäßig $\square$-mittelmäßig $\square$-stark $\square$-äußert

13. Haben Sie während der Stimulation Konzentrationsprobleme gehabt?

\begin{tabular}{|l|l|}
\hline$\square$ ja & $\square$ nein \\
\hline
\end{tabular}

Wenn ja, wie starke Konzentrationsprobleme haben Sie bemerkt?

$\square$-gering $\square$-mäßig $\square$-mittelmäßig $\square$-stark $\square$-äußert 
14. Haben Sie während der Stimulation Sehprobleme gehabt?

\begin{tabular}{|l|l|}
\hline$\square$ ja & $\square$ nein \\
\hline
\end{tabular}

Wenn ja, wie starke Sehprobleme haben Sie bemerkt?
-gering
$\square$-mäßig
-mittelmäßig
$\square$-stark
-äußert

15. Haben Sie während der Stimulation Kopfschmerzen gehabt?

\begin{tabular}{|l|l|}
\hline$\square$ ja & $\square$ nein \\
\hline
\end{tabular}

Wenn ja, wie starke Kopfschmerzen Sie gefühlt haben?

$\square$-gering $\square$-mäßig $\square$-mittelmäßig $\square$-stark $\square$-äußert

16. Haben Sie während der Stimulation etwas Ungewöhnliches gefühlt?

\begin{tabular}{|l|l|}
\hline$\square$ ja & $\square$ nein \\
\hline
\end{tabular}

17. Haben Sie während der Stimulation noch etwas anderes gefühlt?
ja
$\square$ nein

Wenn ja, bitte beschreiben Sie: Bitte hier ausfüllen

18. War die Stimulation für Sie unangenehm?

\begin{tabular}{|l|l|}
\hline ja & $\square$ nein \\
\hline
\end{tabular}

Wenn ja, wie sehr?

$\square$-gering $\square$-mäßig $\square$-mittelmäßig $\square$-stark $\square$-äußert 
19. Haben Sie nach der Stimulation unter den Elektroden Schmerz gefühlt?

\begin{tabular}{|l|l|}
\hline$\square$ ja & $\square$ nein \\
\hline
\end{tabular}

Wenn ja, wie stark?

$\square$-gering $\square$-mäßig $\square$-mittelmäßig $\square$-stark $\square \quad$-nicht aushaltbar

20. Hat ihre Kopfhaut nach der Stimulation unter den Elektroden gekribbelt?

\begin{tabular}{|l|l|}
\hline$\square$ ja & $\square$ nein \\
\hline
\end{tabular}

Wenn ja, wie stark?

$\square$-gering $\square$-mäßig $\square$-mittelmäßig $\square$-stark $\square$-nicht aushaltbar

21. Hat ihre Kopfhaut nach der Stimulation unter den Elektroden gejuckt?

\begin{tabular}{|l|l|}
\hline$\square$ ja & $\square$ nein \\
\hline
\end{tabular}

Wenn ja, wie stark?

$\square$-gering $\square$-mäßig $\square$-mittelmäßig $\square$-stark $\square \quad$-nicht aushaltbar

22. Hat ihre Kopfhaut nach der Stimulation unter den Elektroden gebrannt?

\begin{tabular}{|l|l|}
\hline$\square$ ja & $\square$ nein \\
\hline
\end{tabular}


Wenn ja, wie stark?

$\square$-gering $\square$-mäßig $\square$-mittelmäßig $\square$-stark $\square$-nicht aushaltbar

23. Waren Sie nach der Stimulation müde?

\begin{tabular}{|l|l|}
\hline$\square$ ja & $\square$ nein \\
\hline
\end{tabular}

Wenn ja, wie müde waren Sie?

$\square$-gering $\square$-mäßig $\square$-mittelmäßig $\square$-stark $\square$-äußert

24. Waren Sie nach der Stimulation nervös?

\begin{tabular}{|l|l}
\hline ja & $\square$ nein
\end{tabular}

Wenn ja, wie nervös waren Sie?

$\square$-gering $\square$-mäßig $\square$-mittelmäßig $\square$-stark $\square$-äußert

25. Haben Sie nach der Stimulation Konzentrationsprobleme gehabt?

\begin{tabular}{|l|l|}
\hline$\square$ ja & $\square$ nein \\
\hline
\end{tabular}

Wenn ja, wie starke Konzentrationsprobleme haben Sie bemerkt?
-gering
$\square$-mäßig
$\square$-mittelmäßig
$\square$-stark
-äußert

26. Haben Sie nach der Stimulation Sehprobleme gehabt?

\begin{tabular}{|l|l|}
\hline$\square$ ja & $\square$ nein \\
\hline
\end{tabular}

Wenn ja, wie starke Sehprobleme haben Sie bemerkt?

$\square$-gering $\square$-mäßig $\square$-mittelmäßig $\square$-stark $\square$-äußert 
27. Haben Sie nach der Stimulation Kopfschmerzen gehabt?

\begin{tabular}{|l|l|}
\hline$\square$ ja & $\square$ nein \\
\hline
\end{tabular}

Wenn ja, wie starke Kopfschmerzen Sie gefühlt haben?
-gering
$\square$-mäßig
$\square$-mittelmäßig
$\square$-stark
-äußert

28. War Ihnen übel nach der Stimulation?

\begin{tabular}{|l|l|}
\hline$\square$ ja & $\square$ nein \\
\hline
\end{tabular}

Wenn ja, wie lange war Ihnen übel (in Stunden)? Bitte hier ausfüllen

\section{Haben Sie nach der Stimulation erbrochen?}

\begin{tabular}{|l|l|}
\hline$\square$ ja & $\square$ nein \\
\hline
\end{tabular}

Wenn ja, wie oft? Bitte hier ausfüllen

30. Haben Sie nach der Stimulation Schlafstörungen gehabt?

\begin{tabular}{|l|l|}
\hline$\square$ ja & $\square$ nein \\
\hline
\end{tabular}

Wenn ja, wie viele Tage lang? Bitte hier ausfüllen

31. Haben Sie nach der Stimulation Manie-Symptom (Aufregung) bemerkt?

\begin{tabular}{|l|l|}
\hline$\square$ ja & $\square$ nein \\
\hline
\end{tabular}

Wenn ja, wie lange hat das gedauert (in Stunden)? Bitte hier ausfüllen 
32. War Ihnen nach der Stimulation kalt?

\begin{tabular}{|l|l|}
\hline$\square$ ja & $\square$ nein \\
\hline
\end{tabular}

Wenn ja, wie lange hat das gedauert (in Stunden)? Bitte hier ausfüllen

33. War Ihnen nach der Stimulation warm?

\begin{tabular}{|l|l|}
\hline$\square$ ja & $\square$ nein \\
\hline
\end{tabular}

Wenn ja, wie lange hat das gedauert (in Stunden)? Bitte hier ausfüllen

34. Haben Sie nach der Stimulation etwas Ungewöhnliches gefühlt?

\begin{tabular}{|l|l|}
\hline$\square$ ja & $\square$ nein \\
\hline
\end{tabular}

35. Haben Sie nach der Stimulation noch etwas anderes gefühlt?

\begin{tabular}{|l|l|}
\hline$\square$ ja & $\square$ nein \\
\hline
\end{tabular}

Wenn ja, bitte beschreiben Sie: Bitte hier ausfüllen

36. Haben Sie einen Unterschied zwischen den Stimulationssorten bemerkt?

\begin{tabular}{|l|l|}
\hline$\square$ ja & $\square$ nein \\
\hline
\end{tabular}

Wenn ja, bitte beschreiben Sie das: Bitte hier ausfüllen 
37. Haben Sie Angst vor der Stimulation gehabt?

\begin{tabular}{|l|l|}
\hline$\square$ ja & $\square$ nein \\
\hline
\end{tabular}

38. Möchten Sie später erneut eine Untersuchung mit Gleichstromstimulation mitmachen?

\begin{tabular}{|l|l}
\hline ja & $\square$ nein \\
\hline
\end{tabular}

Bitte überprüfen Sie, ob Sie alle Fragen beantwortet haben.

Vielen Dank! 


\section{Danksagung}

Ich möchte mich an dieser Stelle bei Hr. Prof. Dr. med. Paulus und meiner Betreuerin Fr. Prof. Dr. rer. nat. Antal für die freundliche Überlassung des Themas der Dissertation bedanken.

Mein besonderer Dank gilt Fr. Prof. Dr. rer. nat. Antal und ihren Gruppenmitgliedern Csaba Poreisz, Klára Boros und Daniella Terney, die mich während der Arbeit ausgezeichnet betreuten, berieten und in jeder Hinsicht unterstützten. Sie standen mir mit großem Engagement zur Seite und gaben mir viele hilfreiche und wichtige Anregungen.

Bedanken möchte ich mich auch bei den Patienten, die sich zur Teilnahme an dieser Studie bereit erklärt haben und so freundlich waren, ihre Daten bereitzustellen sowie von ihren Erfahrungen zu berichten. 